\title{
Computing and estimating information matrices of weak ARMA models
}

\author{
Y. Boubacar Mainassara ${ }^{\mathrm{a}}$, M. Carbon ${ }^{\mathrm{b}}$, C. Francq ${ }^{\mathrm{a}}$ \\ ${ }^{a}$ Université Lille III, EQUIPPE-GREMARS, BP 60 149, 59653 Villeneuve d'Ascq cedex, \\ France. \\ ${ }^{b}$ Université Rennes 2 et Ensai
}

\begin{abstract}
Numerous time series admit weak autoregressive-moving average (ARMA) representations, in which the errors are uncorrelated but not necessarily independent nor martingale differences. The statistical inference of this general class of models requires the estimation of generalized Fisher information matrices. We give analytic expressions and propose consistent estimators of these matrices, at any point of the parameter space. Our results are illustrated by means of Monte Carlo experiments and by analyzing the dynamics of daily returns and squared daily returns of financial series.
\end{abstract}

Key words: Asymptotic relative efficiency (ARE), Bahadur's slope, Information matrices, Lagrange Multiplier test, Nonlinear processes, Wald test, Weak ARMA models.

\section{Introduction}

The class of the standard ARMA models with independent errors is often judged too restrictive by practitioners, because they are inadequate for time series exhibiting a nonlinear behavior. Even when the independence assumption is relaxed and the errors are only supposed to be martingale differences, the ARMA models remain often unrealistic because such models postulate that the best predictor is a linear function of the past values.

Email addresses: mailto: yacouba.boubacarmainassara@univ-lille3.fr (Y.

Boubacar Mainassara), mailto:carbon.michel@yahoo.ca (M. Carbon),

mailto: christian.francq@univ-lille3.fr (C. Francq) 
The class of the so-called weak ARMA models with uncorrelated but not necessarily independent errors is much more general and accommodates many nonlinear data-generating processes (see Francq, Roy and Zakoïan, 2005, and the references therein).

For standard ARMA models, it is well known that the asymptotic variance of the least squares estimator (LSE) is of the form $\sigma^{2} J_{\theta_{0}}^{-1}$, where $\sigma^{2}$ is the variance of the errors and $J_{\theta_{0}}$ is an information matrix depending on the ARMA parameter $\theta_{0}$ (see e.g. Brockwell and Davis, 1991). For weak ARMA models, the asymptotic variance of the LSE takes the sandwich form $J_{\theta_{0}}^{-1} I_{\theta_{0}} J_{\theta_{0}}^{-1}$ where $I_{\theta_{0}}$ is a second information matrix depending on $\theta_{0}$ and on fourth-order moments of the errors. The estimation of the asymptotic information matrices $J_{\theta_{0}}$ and $I_{\theta_{0}}$ is thus necessary to evaluate the asymptotic accuracy of the LSE of weak ARMA models.

In the framework of (Gaussian) linear processes, the problem of computing the Fisher information matrices and of their inverses has been widely studied. Various expressions of these matrices have been given by Whittle (1953), Siddiqui (1958), Durbin (1959) and Box and Jenkins (1976). McLeod (1984), Klein and Mélard (1990, 2004) and Godolphin and Bane (2006) have given algorithms for their computation. For few particular cases of weak ARMA models, the matrices $I_{\theta_{0}}$ and $J_{\theta_{0}}$ have been computed by Francq and Zakoian (2000, 2007) and Francq, Roy and Zakoian (2005). In all the above-mentioned references, the information matrices are always computed at the true parameter value $\theta_{0}$. For some applications, in particular to determine Bahadur's slopes under alternatives, it is necessary to compute the information matrices at $\theta \neq \theta_{0}$.

The aim of the present paper is to compute and estimate the information matrices $J_{\theta}$ and $I_{\theta}$ at a point $\theta$ which is not necessarily equal to $\theta_{0}$.

The rest of the paper is organized as follows. In Section 2, we present the weak, strong and semi-strong ARMA representations and recall results concerning the estimation of the weak ARMA models. Section 3 displays the main results. We describe how to obtain numerical evaluations of $I_{\theta}$ and $J_{\theta}$, up to some tolerance, and we propose consistent estimators for these information matrices. Section 4 studies the finite sample behavior of the estimators and compare the Bahadur slopes of two versions of the Lagrange multiplier test for testing linear restrictions on $\theta_{0}$. For the latter application, it is necessary to compute $J_{\theta}$ at $\theta \neq \theta_{0}$. Concluding remarks are proposed in Section 5. The proofs of the main results are collected in the appendix. 


\section{Notations}

We first introduce the notions of weak and strong ARMA representations, which differ by the assumptions on the error terms. We then recall results concerning the estimation of the weak ARMA models, and introduce extended information matrices.

\subsection{Strong, semistrong and weak ARMA representations}

For a linear model to be quite general, the error terms must be the linear innovations, which are uncorrelated by construction but are not independent, nor martingale differences, in general. Indeed, the Wold decomposition (see Brockwell and Davis (1991), Section 5.7) stipulates that any purely non deterministic stationary process can be expressed as

$$
X_{t}=\sum_{\ell=0}^{\infty} \varphi_{\ell} \epsilon_{t-\ell}, \quad\left(\epsilon_{t}\right) \sim \mathrm{WN}\left(0, \sigma^{2}\right),
$$

where $\varphi_{0}=1, \sum_{\ell=0}^{\infty} \varphi_{\ell}^{2}<\infty$, and the notation $\left(\epsilon_{t}\right) \sim \mathrm{WN}\left(0, \sigma^{2}\right)$ signifies that the linear innovation process $\left(\epsilon_{t}\right)$ is a weak white noise, that is a stationary sequence of centered and uncorrelated random variables with common variance $\sigma^{2}$. In practice the sequence $\varphi_{\ell}$ is often parameterized by assuming that $X_{t}$ admits an $\operatorname{ARMA}(p, q)$ representation, i.e. that there exist integers $p$ and $q$ and constants $a_{01}, \ldots, a_{0 p}, b_{01}, \ldots, b_{0 q}$, such that

$$
\forall t \in \mathbb{Z}, \quad X_{t}-\sum_{i=1}^{p} a_{0 i} X_{t-i}=\epsilon_{t}+\sum_{j=1}^{q} b_{0 j} \epsilon_{t-j} .
$$

This representation is said to be a weak $\operatorname{ARMA}(p, q)$ representation under the assumption $\left(\epsilon_{t}\right) \sim \mathrm{WN}\left(0, \sigma^{2}\right)$. For the statistical inference of ARMA models, the weak white noise assumption is not sufficient and is often replaced by the strong white noise assumption $\left(\epsilon_{t}\right) \sim \operatorname{IID}\left(0, \sigma^{2}\right)$, i.e. the assumption that $\left(\epsilon_{t}\right)$ is an independent and identically distributed (iid) sequence of random variables with mean 0 and common variance $\sigma^{2}$. Sometimes an intermediate assumption is considered for the noise. The sequence $\left(\epsilon_{t}\right)$ is said to be a semistrong white noise or a martingale-difference white noise, and is denoted by $\left(\epsilon_{t}\right) \sim \operatorname{MD}\left(0, \sigma^{2}\right)$, if $\left(\epsilon_{t}\right)$ is a stationary sequence satisfying $E\left(\epsilon_{t} \mid \epsilon_{u}, u<t\right)=0$ and $\operatorname{Var}\left(\epsilon_{t}\right)=\sigma^{2}$. An ARMA representation (2) will be called strong under the assumption $\left(\epsilon_{t}\right) \sim \operatorname{IID}\left(0, \sigma^{2}\right)$ and semistrong under the assumption $\left(\epsilon_{t}\right) \sim \operatorname{MD}\left(0, \sigma^{2}\right)$. 
Obviously the strong white noise assumption is more restrictive than that of semistrong white noise, and the latter is more restrictive than the weak white noise assumption, because independence entails unpredictability and unpredictability entails uncorrelatedness, but the reverses are not true. Consequently the weak ARMA representation are more general that the semistrong and strong ones, what we schematize by

$$
\{\text { Weak ARMA }\} \supset\{\text { Semistrong ARMA }\} \supset\{\text { Strong ARMA }\} \text {. }
$$

Any process satisfying (1) is the limit, in $L^{2}$ as $n \rightarrow \infty$, of a sequence of processes satisfying weak $\operatorname{ARMA}\left(p_{n}, q_{n}\right)$ representations (see e.g. Francq and Zakoïan, 2005, page 244). In this sense, the subclass of the processes admitting weak $\operatorname{ARMA}\left(p_{n}, q_{n}\right)$ representations is dense in the set of the purely non deterministic stationary processes. Simple illustrations that the last inclusion of (3) is strict are given by the vast class of volatility models. Indeed GARCHtype models are generally martingale differences (because financial returns are generally assumed to be unpredictable) but they are not strong noises (in particular, because of the volatility clustering, the squared returns are predictable). Many nonlinear models, such as bilinear or Markov-switching models, illustrate the first inclusion in (3), since they admit weak ARMA representation (see Francq, Roy and Zakoïan, 2005, section 2.3) which are not semistrong, because the best predictor is generally not linear when the data generating process (DGP) is nonlinear. To fix ideas, we give below a simple illustrative example, which was not given by the above-mentioned references.

Example 2.1 (Integer-valued AR(1) and MA(1)). McKenzie (2003) reviews the literature on models for integer-valued time series. Let $\circ$ be the thinning operator defined by

$$
a \circ X=\sum_{i=1}^{X} Y_{j}
$$

where $\left(Y_{j}\right)$ is an iid counting series, independent of the integer-valued random variable $X$, with Bernoulli distribution of parameter $a \in[0,1)$. The integervalued autoregressive (INAR) model of order 1 is given by

$$
\forall t \in \mathbb{Z}, \quad X_{t}=a \circ X_{t-1}+Z_{t}
$$


where $Z_{t}$ is an integer-valued iid sequence, independent of the counting series, with mean $\mu$ and variance $\sigma^{2}$. Clearly the best predictor of $X_{t}$ is linear since $E\left(X_{t} \mid X_{u}, u<t\right)=a X_{t-1}+\mu$. Moreover we have $\operatorname{Var}\left(X_{t} \mid X_{u}, u<t\right)=$ $(1-a) a X_{t-1}+\sigma^{2}$. We thus have the semistrong AR(1) representation

$$
X_{t}=a X_{t-1}+\mu+\epsilon_{t}, \quad\left(\epsilon_{t}\right) \sim \operatorname{IID}\left(0, \frac{a \mu}{1-a}+\sigma^{2}\right) .
$$

Similarly to (4) the integer-valued moving-average INMA(1) is defined by

$$
\forall t \in \mathbb{Z}, \quad X_{t}=Z_{t}+a \circ Z_{t-1} .
$$

Straightforward computations show that $E X_{t}=\mu(1+a), \operatorname{Var}\left(X_{t}\right)=\sigma^{2}+$ $a(1-a) \mu+a^{2} \sigma^{2}$ and $\operatorname{Cov}\left(X_{t}, X_{t-1}\right)=a \sigma^{2}$, from which we deduce the weak $M A(1)$ representation

$$
X_{t}=\mu(1+a)+\epsilon_{t}+b \epsilon_{t-1}, \quad\left(\epsilon_{t}\right) \sim \mathrm{WN}\left(0, \sigma_{\epsilon}^{2}\right),
$$

where $b \in[0,1)$ and $\sigma_{\epsilon}^{2}>0$ are solutions of $b /\left(1+b^{2}\right)=\rho_{X}(1)$ and $\left(1+b^{2}\right) \sigma_{\epsilon}^{2}=\operatorname{Var}\left(X_{t}\right)$. This $M A(1)$ representation is not semistrong because $E\left(X_{t} \mid X_{t-1}=0\right)=E\left(X_{t} \mid Z_{t-1}=0\right)=\mu$ does not coincide with the linear prediction given by the $M A(1)$ model when $a \neq 0$.

Finally we have shown that an INMA(1) is a weak MA(1) and that a $\operatorname{INAR(1)}$ is a semistrong $A R(1)$.

\subsection{Estimating weak ARMA representations}

We now present the asymptotic behavior of the LSE in the case of weak ARMA models. The LSE is the standard estimation procedure for ARMA models and it coincides with the maximum-likelihood estimator in the Gaussian case. It will be convenient to write $(2)$ as $\phi_{0}(B) X_{t}=\psi_{0}(B) \epsilon_{t}$, where $B$ is the backshift operator, $\phi_{0}(z)=1-\sum_{i=1}^{p} a_{0 i} z^{i}$ is the AR polynomial and $\psi_{0}(z)=1+\sum_{j=1}^{q} b_{0 j} z^{j}$ is the MA polynomial. The unknown parameter $\theta_{0}=\left(a_{01}, \ldots, a_{0 p}, b_{01}, \ldots, b_{0 q}\right)$ is supposed to belong to the interior of a compact subspace $\Theta^{*}$ of the parameter space

$$
\begin{aligned}
& \Theta:=\left\{\theta=\left(\theta_{1}, \ldots, \theta_{p+q}\right)=\left(a_{1}, \ldots, a_{p}, b_{1}, \ldots, b_{q}\right) \in \mathbb{R}^{p+q}:\right. \\
& \phi(z)=1-\sum_{i=1}^{p} a_{i} z^{i} \text { and } \psi(z)=1+\sum_{i=1}^{q} b_{i} z^{i}
\end{aligned}
$$

have all their zeros outside the unit disk . 
Since $\theta \in \Theta$, the polynomials $\phi_{0}(z)$ and $\psi_{0}(z)$ have all their zeros outside the unit disk. We also assume that $\phi_{0}(z)$ and $\psi_{0}(z)$ have no zero in common, that $p+q>0$ and $a_{0 p}^{2}+b_{0 q}^{2} \neq 0$ (by convention $a_{00}=b_{00}=1$ ). These assumptions are standard and are also made for the usual strong ARMA models.

For all $\theta \in \Theta$, let

$$
\epsilon_{t}(\theta)=\psi^{-1}(B) \phi(B) X_{t}=X_{t}+\sum_{i=1}^{\infty} c_{i}(\theta) X_{t-i} .
$$

Given a realization of length $n, X_{1}, X_{2}, \ldots, X_{n}, \epsilon_{t}(\theta)$ can be approximated, for $0<t \leq n$, by $e_{t}(\theta)$ defined recursively by

$$
e_{t}(\theta)=X_{t}-\sum_{i=1}^{p} \theta_{i} X_{t-i}-\sum_{i=1}^{q} \theta_{p+i} e_{t-i}(\theta)
$$

where the unknown starting values are set to zero: $e_{0}(\theta)=e_{-1}(\theta)=\ldots=$ $e_{-q+1}(\theta)=X_{0}=X_{-1}=\ldots=X_{-p+1}=0$. The random variable $\hat{\theta}_{n}$ is called LSE if it satisfies, almost surely,

$$
Q_{n}\left(\hat{\theta}_{n}\right)=\min _{\theta \in \Theta^{*}} Q_{n}(\theta), \quad Q_{n}(\theta)=\frac{1}{2 n} \sum_{t=1}^{n} e_{t}^{2}(\theta) .
$$

The asymptotic behavior of the LSE is well known in the strong ARMA case, i.e. under the assumption $\left(\epsilon_{t}\right) \sim \operatorname{IID}\left(0, \sigma^{2}\right)$. This assumption being very restrictive, Francq and Zakoïan (1998) considered weak ARMA representations of stationary processes satisfying the following assumption.

A1 : $E\left|X_{t}\right|^{4+2 \nu}<\infty$ and $\sum_{k=0}^{\infty}\left\{\alpha_{X}(k)\right\}^{\frac{\nu}{2+\nu}}<\infty$ for some $\nu>0$,

where $\alpha_{X}(k), k=0,1, \ldots$, denote the strong mixing coefficients of the process $\left(X_{t}\right)$ (see e.g. Bradley, 2005, for a review on strong mixing conditions). As noted by Francq and Zakoïan (2005), Assumption A1 can be replaced by

$\mathbf{A 1}^{\prime}: E\left|\epsilon_{t}\right|^{4+2 \nu}<\infty$ and $\sum_{k=0}^{\infty}\left\{\alpha_{\epsilon}(k)\right\}^{\frac{\nu}{2+\nu}}<\infty$ for some $\nu>0$.

A straightforward extension of Francq and Zakoïan (1998) thus gives the following result. 
Lemma 2.1 (Francq and Zakoïan, 1998). Let $\left(X_{t}\right)$ be a strictly stationary and ergodic process satisfying the weak ARMA model (2) with $\left(\epsilon_{t}\right) \sim$ $\mathrm{WN}\left(0, \sigma^{2}\right)$. Under the previous assumptions and Assumption $\mathbf{A 1}$ or A1',

$$
\sqrt{n}\left(\hat{\theta}_{n}-\theta_{0}\right) \stackrel{d}{\rightarrow} \mathcal{N}\left(0, \Omega=J^{-1} I J^{-1}\right) \text { as } n \rightarrow \infty,
$$

where $I=I_{\theta_{0}}, J=J_{\theta_{0}}=J_{\theta_{0}}^{*}$, with

$$
\begin{aligned}
& I_{\theta}=\sum_{h=-\infty}^{+\infty} \operatorname{Cov}\left\{\epsilon_{t}(\theta) \frac{\partial \epsilon_{t}(\theta)}{\partial \theta}, \epsilon_{t-h}(\theta) \frac{\partial \epsilon_{t-h}(\theta)}{\partial \theta^{\prime}}\right\} \\
& J_{\theta}=E \frac{\partial \epsilon_{t}(\theta)}{\partial \theta} \frac{\partial \epsilon_{t}(\theta)}{\partial \theta^{\prime}}, \quad J_{\theta}^{*}=E \epsilon_{t}(\theta) \frac{\partial^{2} \epsilon_{t}(\theta)}{\partial \theta \partial \theta^{\prime}}+E \frac{\partial \epsilon_{t}(\theta)}{\partial \theta} \frac{\partial \epsilon_{t}(\theta)}{\partial \theta^{\prime}} .
\end{aligned}
$$

In the strong ARMA case, we have $I=I_{s}:=\sigma^{2} J$ and $\Omega=\Omega_{s}:=\sigma^{2} J^{-1}$. In the semistrong ARMA case, i.e. under the assumption $\left(\epsilon_{t}\right) \sim \operatorname{MD}\left(0, \sigma^{2}\right)$, we have

$$
I=I_{s s}:=E \epsilon_{t}^{2} \frac{\partial \epsilon_{t}\left(\theta_{0}\right)}{\partial \theta} \frac{\partial \epsilon_{t}\left(\theta_{0}\right)}{\partial \theta^{\prime}} .
$$

Note that we introduce the two versions $J_{\theta}$ and $J_{\theta}^{*}$ because the following two estimators of $J$ can be considered

$$
\hat{J}_{n}=\frac{1}{n} \sum_{t=1}^{n} \frac{\partial e_{t}\left(\hat{\theta}_{n}\right)}{\partial \theta} \frac{\partial e_{t}\left(\hat{\theta}_{n}\right)}{\partial \theta^{\prime}}, \quad \hat{J}_{n}^{*}=\frac{1}{n} \sum_{t=1}^{n} e_{t}\left(\hat{\theta}_{n}\right) \frac{\partial^{2} e_{t}\left(\hat{\theta}_{n}\right)}{\partial \theta \partial \theta^{\prime}}+\hat{J}_{n} .
$$

The matrices $J_{\theta}, J_{\theta}^{*}$ and $I_{\theta}$ can be called information matrices. As we will see in Section 4.2 they determine the asymptotic behavior of test procedures on $\theta_{0}$. They are also involved in other inference steps, such as in portmanteau adequacy tests (see Francq, Roy and Zakoian, 2005).

\section{Main results}

McLeod (1978) gave a nice expression for $J$, as the variance of a VAR model involving only the ARMA parameter $\theta_{0}$ (see (8.8.3) in Brockwell and Davis, 1991). Francq, Roy and Zakoïan (2005) obtained an expression of $I$ involving the ARMA parameter $\theta_{0}$ and the fourth-order moments of the weak noise $\left(\epsilon_{t}\right)$ (with their notations, $J=\boldsymbol{\Lambda}_{\infty}^{\prime} \boldsymbol{\Lambda}_{\infty}$ and $I=\boldsymbol{\Lambda}_{\infty}^{\prime} \boldsymbol{\Gamma}_{\infty, \infty} \boldsymbol{\Lambda}_{\infty}$ where $\boldsymbol{\Lambda}_{\infty}$ depends on $\theta_{0}$ and $\boldsymbol{\Gamma}_{\infty, \infty}$ depends on moments of $\left.\left(\epsilon_{t}\right)\right)$. For certain statistical applications, it is interesting to obtain similar expressions for $I_{\theta}, J_{\theta}$ and $J_{\theta}^{*}$ when $\theta \neq \theta_{0}$. This is the subject of the next subsection. 
3.1. Theoretical expressions for the information matrices

3.1.1. Matrix $J_{\theta}$

Differentiating the two sides of the equation $\phi(B) X_{t}=\psi(B) \epsilon_{t}(\theta)$, for $i, k=1, \ldots, p$ and $j, \ell=1, \ldots, q$, we obtain

$$
\begin{aligned}
& -X_{t-i}=\psi(B) \frac{\partial}{\partial a_{i}} \epsilon_{t}(\theta), \quad 0=\epsilon_{t-j}(\theta)+\psi(B) \frac{\partial}{\partial b_{j}} \epsilon_{t}(\theta) \\
& 0=\psi(B) \frac{\partial^{2}}{\partial a_{i} \partial a_{k}} \epsilon_{t}(\theta), \quad 0=\frac{\partial}{\partial a_{i}} \epsilon_{t-j}(\theta)+\psi(B) \frac{\partial^{2}}{\partial b_{j} \partial a_{i}} \epsilon_{t}(\theta) \\
& 0=\frac{\partial}{\partial b_{\ell}} \epsilon_{t-j}(\theta)+\frac{\partial}{\partial b_{j}} \epsilon_{t-\ell}(\theta)+\psi(B) \frac{\partial^{2}}{\partial b_{j} \partial b_{\ell}} \epsilon_{t}(\theta) .
\end{aligned}
$$

We thus have

$$
\begin{aligned}
\frac{\partial}{\partial a_{i}} \epsilon_{t}(\theta) & =-\psi^{-1}(B) X_{t-i}=-\psi^{-1} \phi_{0}^{-1} \psi_{0}(B) \epsilon_{t-i}:=-\sum_{h=0}^{\infty} c_{h}^{a} \epsilon_{t-i-h} \\
\frac{\partial}{\partial b_{j}} \epsilon_{t}(\theta) & =-\psi^{-2} \phi_{0}^{-1} \phi \psi_{0}(B) \epsilon_{t-j}:=-\sum_{h=0}^{\infty} c_{h}^{b} \epsilon_{t-j-h}, \\
\frac{\partial^{2}}{\partial b_{j} \partial a_{i}} \epsilon_{t}(\theta) & =\psi^{-2} \phi_{0}^{-1} \psi_{0}(B) \epsilon_{t-i-j}:=\sum_{h=0}^{\infty} c_{h}^{a b} \epsilon_{t-i-j-h}, \\
\frac{\partial^{2}}{\partial b_{j} \partial b_{\ell}} \epsilon_{t}(\theta) & =2 \psi^{-3} \phi_{0}^{-1} \phi \psi_{0}(B) \epsilon_{t-j-\ell}:=\sum_{h=0}^{\infty} c_{h}^{b b} \epsilon_{t-j-\ell-h},
\end{aligned}
$$

and $\partial^{2} \epsilon_{t}(\theta) / \partial a_{i} \partial a_{k}=0$. Moreover

$$
\epsilon_{t}(\theta)=\psi^{-1} \phi_{0}^{-1} \phi \psi_{0}(B) \epsilon_{t}:=\sum_{h=0}^{\infty} c_{h} \epsilon_{t-h} .
$$

The following result immediately follows. 
Proposition 3.1. The elements of the matrix $J_{\theta}$ and $J_{\theta}^{*}$ are given by

$$
\begin{aligned}
J_{\theta}(i, k) & =J_{\theta}^{*}(i, k)=\sigma^{2} \sum_{s=0}^{\infty} c_{s+k-i}^{a} c_{s}^{a}, \\
J_{\theta}(p+j, p+\ell) & =\sigma^{2} \sum_{s=0}^{\infty} c_{s+\ell-j}^{b} c_{s}^{b}, \\
J_{\theta}^{*}(p+j, p+\ell) & =\sigma^{2} \sum_{s=0}^{\infty} c_{s+j+\ell} c_{s}^{b b}+J_{\theta}(p+j, p+\ell), \\
J_{\theta}(i, p+\ell) & =\sigma^{2} \sum_{s=\max \{0, i-\ell\}}^{\infty} c_{s+\ell-i}^{a} c_{s}^{b}, \\
J_{\theta}^{*}(i, p+\ell) & =\sigma^{2} \sum_{s=0}^{\infty} c_{s+i+\ell} c_{s}^{a b}+J_{\theta}(i, p+\ell),
\end{aligned}
$$

for $1 \leq i \leq k \leq p$ and $1 \leq j \leq \ell \leq q$.

On the web page of the authors, programs written in $\mathrm{R}$ are available for computing the information matrices defined in this paper, as well as their estimates. For example, the following function infoJ() computes $J_{\theta}$ when, in $\mathrm{R}$ language, $\theta_{0}<-\mathrm{c}(\operatorname{ar} 0, \mathrm{ma} 0)$ and $\theta<-c(\operatorname{ar} 1, \mathrm{ma} 1)$. The truncation parameter $\mathrm{M}$ is discussed in Section 3.2 below. This function uses the function prod.poly() which makes the product of the 2 polynomials, and the function ARMAtoMA () of the package stats.

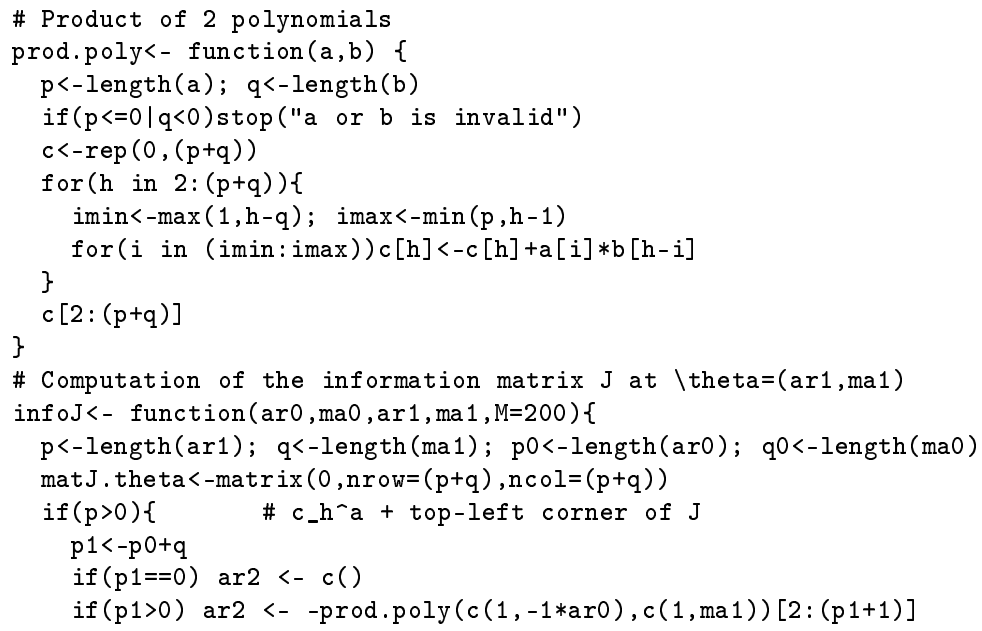




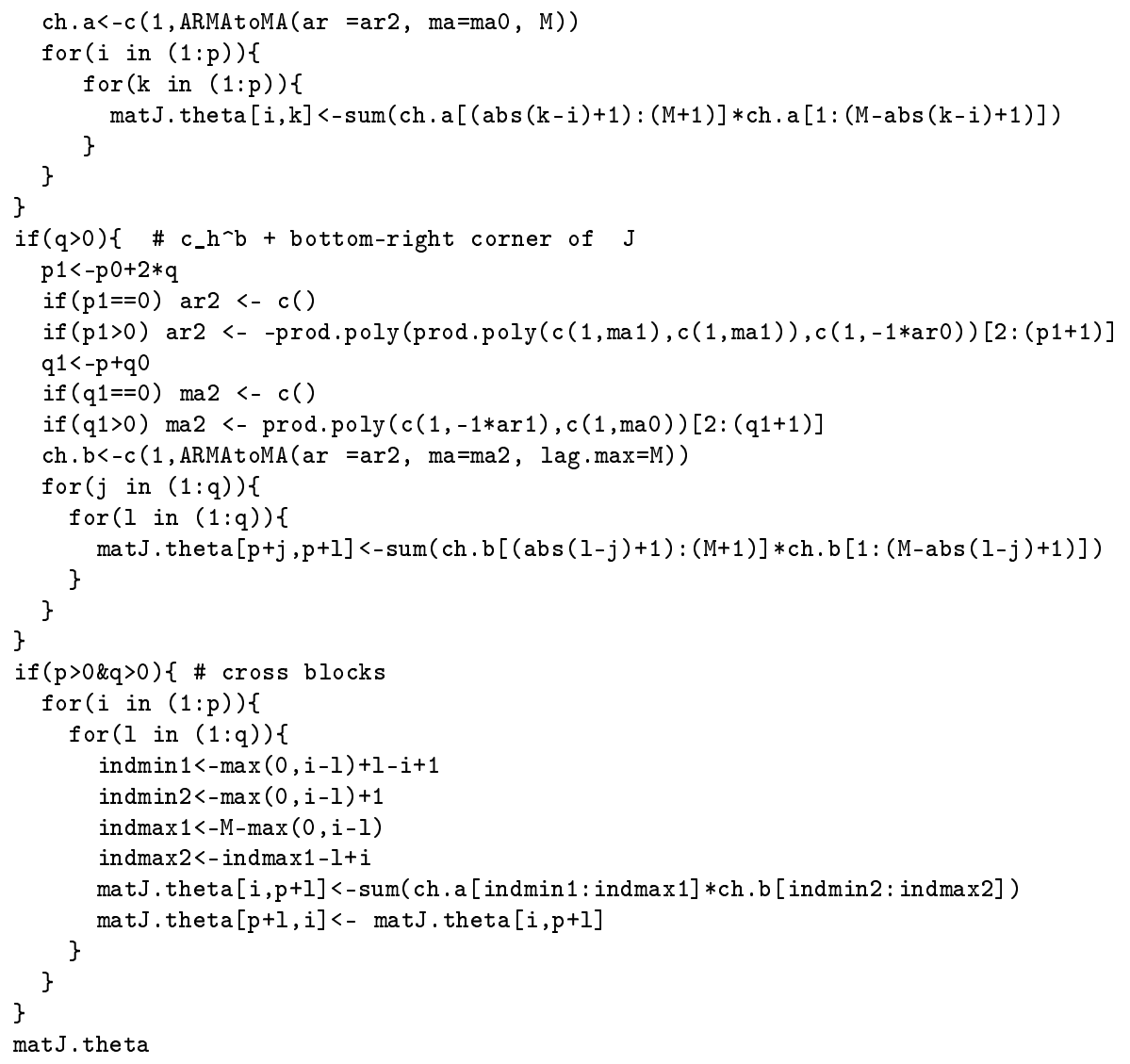

\subsubsection{Matrix $I_{\theta}$}

We now search similar tractable expressions for $I_{\theta}$. Let

$$
\Gamma\left(m, m^{\prime}\right)=\sum_{h=-\infty}^{+\infty} \operatorname{Cov}\left(\epsilon_{t} \epsilon_{t-m}, \epsilon_{h} \epsilon_{h-m^{\prime}}\right) .
$$

In the strong case, we have

$$
\Gamma(0,0)=\mu_{4}-\sigma^{4}, \quad \Gamma(m, m)=\Gamma(m,-m)=\sigma^{4}, \quad \Gamma\left(m^{\prime}, m^{\prime \prime}\right)=0,
$$

with $\mu_{4}=E \epsilon_{1}^{4}, m \neq 0$ and $\left|m^{\prime}\right| \neq\left|m^{\prime \prime}\right|$. Simplifications may also hold in semistrong cases. Indeed, consider the case $\left(\epsilon_{t}\right) \sim \mathrm{WN}\left(0, \sigma_{\epsilon}^{2}\right)$ under the following symmetry assumption

$$
E \epsilon_{t_{1}} \epsilon_{t_{2}} \epsilon_{t_{3}} \epsilon_{t_{4}}=0 \quad \text { when } \quad t_{1} \neq t_{2}, t_{1} \neq t_{3} \text { and } t_{1} \neq t_{4} .
$$


A similar assumption is made in Francq and Zakoian (2009b). In this paper, it is shown that, in particular, GARCH models with fourth-order moments and symmetric innovations satisfy (9). Many other martingale differences satisfy this assumption. In this semistrong case, we have

$$
\Gamma(0,0)=\sum_{h=-\infty}^{\infty} \operatorname{Cov}\left(\epsilon_{t}^{2}, \epsilon_{t-h}^{2}\right), \quad \Gamma(m, m)=E \epsilon_{t}^{2} \epsilon_{t-m}^{2}, \quad \Gamma\left(m^{\prime}, m^{\prime \prime}\right)=0
$$

when $m \neq 0$ and $\left|m^{\prime}\right| \neq\left|m^{\prime \prime}\right|$.

Example 3.1. For a GARCH(1,1) model of the form

$$
\left\{\begin{array}{l}
\epsilon_{t}=\sqrt{h_{t}} \eta_{t}, \quad t=1,2, \ldots \\
h_{t}=\omega+\alpha \epsilon_{t-1}^{2}+\beta h_{t-1}, \quad\left(\eta_{t}\right) \sim \operatorname{IID}(0,1)
\end{array}\right.
$$

with $\omega>0, \alpha \geq 0, \beta \geq 0$ and $\alpha^{2} E \eta_{1}^{4}+\beta^{2}+2 \alpha \beta<1^{1}$ we obtain

$$
\begin{aligned}
\Gamma(0,0) & =E \nu_{t}^{2} \frac{(1-\beta)^{2}}{(1-\alpha-\beta)^{2}}, \\
\Gamma(1,1) & =E \nu_{t}^{2}\left(\alpha+\frac{\alpha^{2}(\alpha+\beta)}{1-(\alpha+\beta)^{2}}\right)+\left(E \sigma_{t}^{2}\right)^{2} \\
\Gamma(m, m) & =(\alpha+\beta) \Gamma(m-1, m-1)+\omega E \sigma_{t}^{2}, \quad m>1,
\end{aligned}
$$

with $E \nu_{t}^{2}=E \eta_{1}^{4}\left(E \sigma_{t}^{4}+1-2 E \sigma_{t}^{2}\right)$,

$$
E \sigma_{t}^{2}=\frac{\omega}{1-\alpha-\beta}, \quad E \sigma_{t}^{4}=\frac{\omega^{2}(1+\alpha+\beta)}{\left(1-\alpha^{2} E \eta_{1}^{4}-\beta^{2}-2 \alpha \beta\right)(1-\alpha-\beta)} .
$$

Proposition 3.2. The elements of the matrix $I_{\theta}$ are given by

$$
\begin{aligned}
I_{\theta}(i, k) & =\sum_{h_{1}, h_{2}, h_{3}, h_{4}=0}^{+\infty} c_{h_{1}} c_{h_{2}}^{a} c_{h_{3}} c_{h_{4}}^{a} \Gamma\left(h_{2}+i-h_{1}, h_{4}+k-h_{3}\right), \\
I_{\theta}(j, \ell) & =\sum_{h_{1}, h_{2}, h_{3}, h_{4}=0}^{+\infty} c_{h_{1}} c_{h_{2}}^{b} c_{h_{3}} c_{h_{4}}^{b} \Gamma\left(h_{2}+j-h_{1}, h_{4}+\ell-h_{3}\right), \\
I_{\theta}(i, \ell) & =\sum_{h_{1}, h_{2}, h_{3}, h_{4}=0}^{+\infty} c_{h_{1}} c_{h_{2}}^{a} c_{h_{3}} c_{h_{4}}^{b} \Gamma\left(h_{2}+i-h_{1}, h_{4}+\ell-h_{3}\right),
\end{aligned}
$$

\footnotetext{
${ }^{1}$ The latter conditions and necessary and sufficient for the existence of a nonanticipative stationary solution with fourth-order moments (see e.g. Example 2.3 in Francq and Zakoian, 2010).
} 
for $1 \leq i \leq k \leq p$ and $1 \leq j \leq \ell \leq q$

Note that $c_{0}=1$ and that, at $\theta=\theta_{0}$, we have $c_{h}=0$ for $h>0$. The expression of $I=I_{\theta_{0}}$ thus simplifies to that given in Francq, Roy and Zakoian (2005). There is also a slight simplification in the strong and semistrong ARMA cases because, in view of (8) and (10), $I_{\theta}$ is then obtained by summing over 3 indices instead of 4 .

\subsubsection{Examples of analytic and numerical computations of $J_{\theta}$ and $I_{\theta}$}

Let us compute the information matrices of an $\operatorname{ARMA}(1,1)$ model at the point $\theta^{*}=(a, 0)^{\prime}$ when $\theta_{0}=\left(0, b_{0}\right)^{\prime}$ (i.e. the DGP is a MA(1)). We have $\epsilon_{t}(\theta)=\sum_{h=0}^{\infty}(-b)^{h}\left(X_{t-h}-a X_{t-h-1}\right)$. It follows that $\epsilon_{t}\left(\theta^{*}\right)=\epsilon_{t}+$ $\left(b_{0}-a\right) \epsilon_{t-1}-a b_{0} \epsilon_{t-2}, \partial \epsilon_{t}\left(\theta^{*}\right) / \partial a=-\epsilon_{t-1}-b_{0} \epsilon_{t-2}, \partial \epsilon_{t}\left(\theta^{*}\right) / \partial b=-\epsilon_{t-1}-$ $\left(b_{0}-a\right) \epsilon_{t-2}+a b_{0} \epsilon_{t-3}, \partial^{2} \epsilon_{t}\left(\theta^{*}\right) / \partial a^{2}=0, \partial^{2} \epsilon_{t}\left(\theta^{*}\right) / \partial a \partial b=\epsilon_{t-2}+b_{0} \epsilon_{t-3}$ and $\partial^{2} \epsilon_{t}\left(\theta^{*}\right) / \partial b^{2}=2 \epsilon_{t-2}+2\left(b_{0}-a\right) \epsilon_{t-3}-2 a b_{0} \epsilon_{t-4}$.

Thus

$$
\begin{aligned}
J_{\theta^{*}} & =\sigma^{2}\left(\begin{array}{cc}
1+b_{0}^{2} & 1+b_{0}\left(b_{0}-a\right) \\
1+b_{0}\left(b_{0}-a\right) & 1+\left(b_{0}-a\right)^{2}+a^{2} b_{0}^{2}
\end{array}\right), \\
J_{\theta^{*}}^{*} & =J_{\theta^{*}}+\sigma^{2}\left(\begin{array}{cc}
0 & -a b_{0} \\
-a b_{0} & -2 a b_{0}
\end{array}\right) .
\end{aligned}
$$

Now assume that $\epsilon_{t}$ is the weak white noise considered by Romano and Thombs (1996), defined by

$$
\epsilon_{t}=\eta_{t} \eta_{t-1} \cdots \eta_{t-k}, \quad\left(\eta_{t}\right) \sim \operatorname{IID} \mathcal{N}(0,1), \quad k \geq 0
$$

It seems impossible to obtain $I_{\theta^{*}}$ explicitly, but the information matrices can be obtained explicitly at $\theta_{0}$ :

$$
\begin{aligned}
& J_{\theta_{0}}=J_{\theta_{0}}^{*}=\left(\begin{array}{cc}
1 & 1 \\
1 & 1 /\left(1-b_{0}^{2}\right)
\end{array}\right) \\
& I_{\theta_{0}}=3^{k}\left(\begin{array}{cc}
1 & 1 \\
1 & \frac{1-\left(b^{2} / 3\right)^{k+1}}{1-b^{2} / 3}+\frac{b^{2(k+1)}}{3^{k}\left(1-b^{2}\right)}
\end{array}\right) .
\end{aligned}
$$

Note that $I_{\theta_{0}}=J_{\theta_{0}}$ in the strong case (i.e. when $k=0$ ). For more complex models or at some point $\theta^{*} \neq \theta_{0}$ the evaluation of these information matrices is not feasible analytically but they can be easily obtained numerically. For 
instance, on this example with $k=3, \theta_{0}=(0,0.5)^{\prime}$ and $\theta^{*}=(-0.4,-0.5)^{\prime}$, we have

$$
J_{\theta^{*}}=\left(\begin{array}{cc}
2.33 & 4.33 \\
4.33 & 11.25
\end{array}\right), J_{\theta^{*}}^{*}=\left(\begin{array}{cc}
2.33 & 6.33 \\
6.33 & 17.65
\end{array}\right), I_{\theta^{*}}=\left(\begin{array}{cc}
1161.92 & 2177.66 \\
2177.66 & 4187.63
\end{array}\right) .
$$

\subsection{Approximation of the information matrices by finite sums}

In practice the infinite sums involved in $J_{\theta}, J_{\theta}^{*}$ and $I_{\theta}$ are truncated. This section concentrates on the choice of the truncation parameter for $I_{\theta}$, the problem being similar, and somewhat simpler, for the other matrices. Matrix $I_{\theta}$ is truncated by the matrix $I_{\theta}^{M}$ of $M^{4}$ terms, defined by

$$
I_{\theta}^{M}(i, k)=\sum_{h_{1}, h_{2}, h_{3}, h_{4}=0}^{M} c_{h_{1}} c_{h_{2}}^{a} c_{h_{3}} c_{h_{4}}^{a} \Gamma\left(h_{2}+i-h_{1}, h_{4}+k-h_{3}\right),
$$

when $1 \leq i \leq k \leq p$, and whose the other elements are defined similarly. The following proposition defines a value of $M$ such that $I_{\theta}^{M}$ be equal to $I_{\theta}$ up to an arbitrarily small tolerance number $\varepsilon$. Let the matrix norm defined by $\|A\|=\sum_{i, j}|A(i, j)|$ with obvious notations.

Proposition 3.3. Let $\bar{\rho}$ be the inverse of the largest modulus of the zeroes of the polynomials $\phi_{0}$ and $\psi$, let

$$
\bar{\Gamma}=\max _{m, m^{\prime} \geq 0}\left|\Gamma\left(m, m^{\prime}\right)\right|, \quad \bar{\pi}=\left(1+\sum_{j=1}^{q}\left|b_{0 j}\right|\right) \max _{i=0, \ldots, p}\left|a_{i}\right|
$$

with $\left|a_{0}\right|=1$, and let

$$
K=\sqrt{2}(p+q+1) \bar{\pi} \bar{\Gamma}^{1 / 4}\left(\frac{-2(p+2 q-1)}{\log \bar{\rho}}\right)^{(p+2 q-1)} \bar{\rho}^{-0.5-(p+2 q-1) / \log \bar{\rho}} .
$$

For all $\varepsilon>0$, if

$$
M \geq M_{\epsilon}:=\frac{\log \sqrt{\varepsilon}(1-\sqrt{\log \bar{\rho}})^{2} / K^{2}}{\log \bar{\rho}}
$$

then

$$
\left\|I_{\theta}-I_{\theta}^{M}\right\| \leq(p+q)^{2} \varepsilon
$$




\subsection{Estimation of the information matrices}

Let $J_{\theta, n}$ and $J_{\theta, n}^{*}$ be defined as in (6), replacing $\hat{\theta}_{n}$ by $\theta$ in $\hat{J}_{n}$ and $\hat{J}_{n}^{*}$, so that $\hat{J}_{n}=J_{\hat{\theta}_{n}, n}$ and $\hat{J}_{n}^{*}=J_{\hat{\theta}_{n}, n}^{*}$. The following result shows that an estimator of $J_{\theta^{*}}$ is trivially deduced from one of $\theta^{*}$.

Proposition 3.4. Under the assumptions of Lemma 2.1, as $n \rightarrow \infty$,

$$
\text { if } \theta_{n}^{*} \rightarrow \theta^{*} \text { a.s. then } J_{\theta_{n}^{*}, n} \rightarrow J_{\theta^{*}} \text { and } J_{\theta_{n}^{*}, n}^{*} \rightarrow J_{\theta^{*}}^{*} \text { a.s. }
$$

The estimation of the long-run variance $I_{\theta}$ is more complicated. In the literature, two types of estimators are generally employed: Heteroskedasticity and Autocorrelation Consistent (HAC) estimators (see Newey and West (1987) and Andrews (1991) for general references, and Francq and Zakoian (2007) for an application to testing strong linearity in weak ARMA models) and spectral density estimators (see e.g. den Haan and Levin (1997) for a general reference and Francq, Roy and Zakoian (2005) for estimating $I$ in the present context). We will extend the results of Francq, Roy and Zakoian (2005) for estimating $I_{\theta}$ when $\theta$ is not necessarily equal to $\theta_{0}$.

\subsubsection{An estimator based on a spectral density form for $I_{\theta}$}

Note $(2 \pi)^{-1} I_{\theta^{*}}$ is the spectral density at frequency 0 (see Brockwell and Davis (1991) p. 459) of the process

$$
\nabla_{t}=S_{t}-E S_{t}, \quad S_{t}=\epsilon_{t}\left(\theta^{*}\right) \frac{\partial \epsilon_{t}\left(\theta^{*}\right)}{\partial \theta} .
$$

For any given $\theta^{*} \in \Theta, S_{t}$ is a measurable function of $\left\{X_{u}, u \leq t\right\}$. Let $\hat{S}_{t}$ be obtained by replacing the unknown initial values $\left\{X_{u}, u \leq 0\right\}$ by 0 and $\theta^{*}$ by $\theta_{n}^{*}$ in $S_{t}$. Let also

$$
\hat{\nabla}_{t}=\hat{S}_{t}-\frac{1}{n} \sum_{t=1}^{n} \hat{S}_{t} .
$$

The stationary process $\left(\nabla_{t}\right)$ admits the Wold decomposition $\nabla_{t}=u_{t}+$ $\sum_{i=1}^{\infty} B_{i} u_{t-i}$, where $\left(u_{t}\right)$ is a $(p+q)$-variate weak white noise with covariance matrix $\Sigma_{u}$. Assume that $\Sigma_{u}$ is non-singular, that $\sum_{i=1}^{\infty}\left\|B_{i}\right\|<\infty$, and that $\operatorname{det}\left(I_{p+q}+\sum_{i=1}^{\infty} B_{i} z^{i}\right) \neq 0$ when $|z| \leq 1$. Then $\left(\nabla_{t}\right)$ admits an $\operatorname{AR}(\infty)$ representation of the form

$$
\mathcal{A}(B) \nabla_{t}:=\nabla_{t}-\sum_{i=1}^{\infty} A_{i} \nabla_{t-i}=u_{t}
$$


such that $\sum_{i=1}^{\infty}\left\|A_{i}\right\|<\infty$ and $\operatorname{det}\{\mathcal{A}(z)\} \neq 0$ for all $|z| \leq 1$, and we obtain

$$
I_{\theta}=\mathcal{A}^{-1}(1) \Sigma_{u} \mathcal{A}^{\prime-1}(1) .
$$

In the framework of univariate linear processes with independent innovations, Berk (1974) showed that the spectral density can be consistently estimated by fitting autoregressive models of order $r=r(n)$, whenever $r \rightarrow \infty$ and $r^{3} / n \rightarrow 0$ as $n \rightarrow \infty$. It can be shown that this result remains valid for the multivariate linear process $\left(\nabla_{t}\right)$, though its innovation $\left(u_{t}\right)$ is not an independent process. Another difference with Berk (1974), is that $\left(\nabla_{t}\right)$ is not directly observed and is replaced by $\left(\hat{\nabla}_{t}\right)$.

Consider the regression of $\nabla_{t}$ on $\nabla_{t-1}, \ldots, \nabla_{t-r}$ defined by

$$
\nabla_{t}=\sum_{i=1}^{r} A_{r, i} \nabla_{t-i}+u_{r, t}, \quad u_{r, t} \perp\left\{\nabla_{t-1} \cdots \nabla_{t-r}\right\}
$$

The least squares estimators of $\underline{A}_{r}=\left(A_{r, 1} \cdots A_{r, r}\right)$ and $\Sigma_{u_{r}}=\operatorname{Var}\left(u_{r, t}\right)$ are defined by

$$
\underline{\hat{A}}_{r}=\hat{\Sigma}_{\hat{\nabla}, \hat{\underline{\Sigma}}_{r}} \hat{\Sigma}_{\hat{\underline{V}}_{r}}^{-1} \quad \text { and } \quad \hat{\Sigma}_{u_{r}}=\frac{1}{n} \sum_{t=1}^{n}\left(\hat{\nabla}_{t}-\underline{\hat{A}}_{r} \underline{\hat{\nabla}}_{r, t}\right)\left(\hat{\nabla}_{t}-\underline{\hat{A}}_{r} \underline{\hat{\nabla}}_{r, t}\right)^{\prime}
$$

where $\underline{\nabla}_{r, t}=\left(\hat{\nabla}_{t-1}^{\prime} \cdots \hat{\nabla}_{t-r}^{\prime}\right)^{\prime}$,

$$
\hat{\Sigma}_{\hat{\nabla}, \hat{\nabla}_{r}}=\frac{1}{n} \sum_{t=1}^{n} \hat{\nabla}_{t} \underline{\hat{\nabla}}_{r, t}^{\prime}, \quad \hat{\Sigma}_{\hat{\vec{V}}_{r}}=\frac{1}{n} \sum_{t=1}^{n} \underline{\hat{\nabla}}_{r, t} \underline{\hat{\nabla}}_{r, t}^{\prime},
$$

with by convention $\hat{\nabla}_{t}=0$ when $t \leq 0$, and assuming $\hat{\Sigma}_{\hat{\vec{V}}_{r}}$ is non singular (which holds true asymptotically).

Under mild regularity conditions (the precise statement of the result and its proof are available from the authors under request), it can be shown that if $\theta_{n}^{*} \rightarrow \theta^{*}$ almost surely,

$$
I_{\theta_{n}^{*}, n}=\hat{\mathcal{A}}_{r}^{-1}(1) \hat{\Sigma}_{u_{r}} \hat{\mathcal{A}}_{r}^{\prime-1}(1) \rightarrow I_{\theta^{*}}
$$

in probability when $r=r(n) \rightarrow \infty$ and $r^{3} / n \rightarrow 0$ as $n \rightarrow \infty$.

For the implementation of $I_{\theta_{n}^{*}, n}, \operatorname{AR}(r)$ models are obtained recursively for $r=0,1, \ldots, r_{\max }$ (with $r_{\max }=15$ for the forthcoming applications), using the efficient Whittle's (1963) generalization of the Durbin-Levinson algorithm, described for instance in Brockwell and Davis (1991) Theorem 5.2.1. The order $r$ is then selected using the AIC criterion. 


\section{Applications}

A first set of experiments illustrates the finite sample behavior of our estimators of the information matrices $I_{\theta^{*}}, J_{\theta^{*}}$ and $J_{\theta^{*}}^{*}$, for strong and weak ARMA models. We then study the impact of the estimator of $J$, i.e. the effect of choosing $\hat{J}_{n}$ or $\hat{J}_{n}^{*}$ defined by (6), on the asymptotic behavior of tests of linear restrictions on the ARMA parameters. For this study it will be necessary to evaluate $J_{\theta^{*}}$ at $\theta^{*} \neq \theta_{0}$. Finally, an application to financial data is presented.

\subsection{Finite sample behavior of estimators of the information matrices}

To investigate the finite sample performance of the estimators, we simulated $N=1,000$ independent trajectories of size $n=1,000$ and $n=10,000$ of an $\operatorname{ARMA}(1,1)$ model with parameter $\theta_{0}=(0.5,0.7)$, in which the noise is defined by (11). Note that when $k=0$ in (11), the ARMA model is strong, whereas the model is weak when $k>0$.

\subsubsection{Estimating the information matrices at a given point $\theta^{*}$}

The information matrices $J_{\theta^{*}}, J_{\theta^{*}}^{*}$ and $I_{\theta^{*}}$ have been computed and estimated at 3 points $\theta^{*}$ chosen randomly in $(-1,1)^{2}$. The estimators are $J_{\theta^{*}, n}$, $J_{\theta^{*}, n}^{*}$ and $I_{\theta^{*}, n}$ defined in Proposition 3.4 and (16). Table 1 displays the average, over the $N$ replications, of the relative estimation errors

$$
\frac{\left\|J_{\theta^{*}, n}-J_{\theta^{*}}\right\|}{\left\|J_{\theta^{*}}\right\|}, \quad \frac{\left\|J_{\theta^{*}, n}^{*}-J_{\theta^{*}}^{*}\right\|}{\left\|J_{\theta^{*}}^{*}\right\|} \text { and } \frac{\left\|I_{\theta^{*}, n}-I_{\theta^{*}}\right\|}{\left\|I_{\theta^{*}}\right\|} .
$$

From Table 1, one can note that: 1) although the information matrices vary a lot with $\theta^{*}$, the relative errors are not very sensitive to the value of $\theta^{*}$; 2 ) as expected the relative errors decrease when $n$ increases; 3 ) it is more difficult to estimate the information matrices when $k$ is large; 4) it is easier to estimate $J_{\theta^{*}}$ than $J_{\theta^{*}}^{*}$, and easier to estimate $J_{\theta^{*}}^{*}$ than $I_{\theta^{*}}$.

\subsubsection{Estimating the asymptotic variance of the LSE}

Several estimators of the asymptotic variance $\Omega$ involved in (5) can be considered. In view of Proposition 3.4 and (16), two estimators that are consistent under very general assumptions are

$$
\hat{\Omega}=J_{\hat{\theta}_{n}, n}^{-1} I_{\hat{\theta}_{n}, n} J_{\hat{\theta}_{n}, n}^{-1} \quad \text { and } \quad \hat{\Omega}^{*}=J_{\hat{\theta}_{n}, n}^{*-1} I_{\hat{\theta}_{n}, n} J_{\hat{\theta}_{n}, n}^{*-1} .
$$


Table 1: Average relative error for the estimators of the information matrices, over $N=1000$ replications.

\begin{tabular}{cccccccc}
\hline \hline & \multicolumn{3}{c}{$n=1,000$} & & \multicolumn{3}{c}{$n=10,000$} \\
$k$ & $\theta^{*}$ & $J_{\theta^{*}}$ & $J_{\theta^{*}}^{*}$ & $I_{\theta^{*}}$ & $J_{\theta^{*}}$ & $J_{\theta^{*}}^{*}$ & $I_{\theta^{*}}$ \\
\hline \multirow{3}{*}{0} & $(-0.9,-0.7)$ & 0.10307 & 0.13466 & 0.34958 & 0.03472 & 0.04466 & 0.13070 \\
& $(0.5,-0.6)$ & 0.07817 & 0.10840 & 0.28935 & 0.02635 & 0.03655 & 0.11602 \\
& $(0.7,0.9)$ & 0.16050 & 0.23238 & 0.62677 & 0.05105 & 0.07239 & 0.35446 \\
\hline \multirow{4}{*}{1} & $(-0.9,-0.7)$ & 0.15183 & 0.17483 & 0.54247 & 0.04835 & 0.05592 & 0.23380 \\
& $(0.5,-0.6)$ & 0.13454 & 0.15691 & 0.52612 & 0.04225 & 0.04979 & 0.22927 \\
& $(0.7,0.9)$ & 0.19607 & 0.25395 & 0.81663 & 0.06119 & 0.08024 & 0.41813 \\
\hline \multirow{3}{*}{2} & $(-0.9,-0.7)$ & 0.24941 & 0.26726 & 1.01021 & 0.07945 & 0.08502 & 0.48073 \\
& $(0.5,-0.6)$ & 0.23188 & 0.25324 & 1.02312 & 0.07372 & 0.08064 & 0.48426 \\
& $(0.7,0.9)$ & 0.25566 & 0.29135 & 0.88026 & 0.08983 & 0.10374 & 0.52578 \\
\hline \multirow{3}{*}{3} & $(-0.9,-0.7)$ & 0.36007 & 0.37685 & 1.13567 & 0.13339 & 0.13941 & 0.80758 \\
& $(0.5,-0.6)$ & 0.34200 & 0.36553 & 1.11668 & 0.12598 & 0.13487 & 0.80077 \\
& $(0.7,0.9)$ & 0.38990 & 0.41912 & 1.35145 & 0.13595 & 0.14663 & 0.76092 \\
\hline \multirow{4}{*}{4} & $(-0.9,-0.7)$ & 0.49452 & 0.50613 & 1.19581 & 0.20527 & 0.21180 & 1.11480 \\
& $(0.5,-0.6)$ & 0.47746 & 0.49784 & 1.19473 & 0.19569 & 0.20733 & 1.10979 \\
& $(0.7,0.9)$ & 0.54117 & 0.55883 & 1.95453 & 0.21114 & 0.21816 & 1.07556 \\
\hline \multirow{2}{*}{5} & $(-0.9,-0.7)$ & 0.67610 & 0.68923 & 1.42507 & 0.31757 & 0.32562 & 1.66401 \\
& $(0.5,-0.6)$ & 0.66101 & 0.68331 & 1.44809 & 0.30515 & 0.32038 & 1.67812 \\
& $(0.7,0.9)$ & 0.69161 & 0.70621 & 1.77970 & 0.29799 & 0.30403 & 1.15614 \\
\hline \hline
\end{tabular}

In view of the consistency of the LSE stated in Lemma 2.1 and Proposition 3.1, the matrix $J$ can be estimated by plugging. We then define the alternative estimator

$$
\Omega\left(\hat{\theta}_{n}\right)=J_{\hat{\theta}_{n}}^{-1} I_{\hat{\theta}_{n}, n} J_{\hat{\theta}_{n}}^{-1} .
$$

Other estimators of $\Omega$ that should be consistent in the strong ARMA case are defined by

$$
\hat{\Omega}_{s}=\hat{\sigma}^{2} J_{\hat{\theta}_{n}, n}^{-1} \quad \text { and } \quad \Omega_{s}\left(\hat{\theta}_{n}\right)=\hat{\sigma}^{2} J_{\hat{\theta}_{n}}^{-1}, \quad \text { with } \quad \hat{\sigma}^{2}=\frac{1}{n} \sum_{t=1}^{n} e_{t}^{2}\left(\hat{\theta}_{n}\right) \text {. }
$$


Finally, in the semi-strong case, an estimator is given by

$$
\hat{\Omega}_{s s}=J_{\hat{\theta}_{n}, n}^{-1} \hat{I}_{s s} J_{\hat{\theta}_{n}, n}^{-1}, \quad \hat{I}_{s s}=\frac{1}{n} \sum_{t=1}^{n} e_{t}^{2}\left(\hat{\theta}_{n}\right) \frac{\partial e_{t}\left(\hat{\theta}_{n}\right)}{\partial \theta} \frac{\partial e_{t}\left(\hat{\theta}_{n}\right)}{\partial \theta^{\prime}} .
$$

Table 2 indicates that, for all the consistent estimators (i.e. all estimators when $k=0$, and $\hat{\Omega}, \hat{\Omega}^{*}, \Omega\left(\hat{\theta}_{n}\right)$ and $\hat{\Omega}_{s s}$ when $\left.k>0\right)$ the relative errors decrease when $n$ increases. As expected from Table 1 , the estimation of the asymptotic matrices becomes more difficult when $k$ increases. When $k>0$ the estimator $\hat{\Omega}_{s s}$ is much more accurate than $\hat{\Omega}_{s}$ and $\Omega_{s}\left(\hat{\theta}_{n}\right)$ (which are actually not consistent in this semi-strong setting) and also slightly more accurate than the other ones. In the strong case (i.e. when $k=0$ in (11)), the estimators $\hat{\Omega}_{s}$ and $\Omega_{s}\left(\hat{\theta}_{n}\right)$ are much more accurate than the other ones, but they are not consistent when $k>0$ (the relative errors are almost the same for $n=1,000$ and $n=10,000)$. This not surprising, because the noise defined in (11) is a semistrong one.

Table 2: Relative error of the asymptotic variance matrices of the LSE. The number of replications is $N=1000$.

\begin{tabular}{cccccccc}
\hline \hline$n$ & $k$ & $\hat{\Omega}$ & $\hat{\Omega}^{*}$ & $\Omega\left(\hat{\theta}_{n}\right)$ & $\hat{\Omega}_{s}$ & $\hat{\Omega}_{s}\left(\hat{\theta}_{n}\right)$ & $\Omega_{s s}$ \\
\hline \multirow{4}{*}{1,000} & 0 & 0.13327 & 0.18264 & 0.15751 & 0.06353 & 0.07496 & 0.09014 \\
& 1 & 0.35768 & 0.36452 & 0.42471 & 0.47470 & 0.48099 & 0.24168 \\
& 2 & 0.52599 & 0.53392 & 0.65840 & 0.79349 & 0.79581 & 0.43806 \\
& 3 & 0.69808 & 0.70883 & 0.84601 & 0.92835 & 0.92945 & 0.62591 \\
& 4 & 0.83908 & 0.85469 & 0.97604 & 0.97497 & 0.97583 & 0.76076 \\
& 5 & 0.92800 & 0.92828 & 0.95288 & 0.99173 & 0.99213 & 0.87238 \\
& 10 & 1.00805 & 0.99961 & 0.99892 & 1.00006 & 0.99983 & 1.02399 \\
\hline \multirow{4}{*}{10,000} & 0 & 0.04344 & 0.05567 & 0.05006 & 0.02002 & 0.02327 & 0.02906 \\
& 1 & 0.14336 & 0.14478 & 0.16351 & 0.47000 & 0.47020 & 0.08734 \\
& 2 & 0.31212 & 0.31412 & 0.35461 & 0.79448 & 0.79442 & 0.23533 \\
& 3 & 0.50658 & 0.51049 & 0.58767 & 0.92943 & 0.92913 & 0.43183 \\
& 4 & 0.65129 & 0.66057 & 0.78561 & 0.97604 & 0.97626 & 0.62521 \\
& 5 & 0.76824 & 0.77414 & 0.88845 & 0.99198 & 0.99212 & 0.72576 \\
& 10 & 0.99694 & 0.99672 & 0.99761 & 0.99996 & 0.99996 & 0.99331 \\
\hline \hline
\end{tabular}




\subsection{Bahadur's slopes of two versions of the Lagrange-Multiplier test}

Let $R$ be a given matrix of size $s_{0} \times(p+q)$ and rank $s_{0}$, and let $r_{0}$ and $r_{1}$ be given vectors of size $s_{0}$ such that $r_{1} \neq r_{0}$. Consider the testing problem

$$
H_{0}: R \theta_{0}=r_{0} \quad \text { against } \quad H_{1}: R \theta_{0}=r_{1} .
$$

When the model is much simpler under the null than under the alternative, the Lagrange-Multiplier (LM) test is very attractive because, contrary to other tests, in particular the Wald and Likelihood-Ratio tests, the LM procedure only requires the estimation of the ARMA model under $H_{0}$ (see Engle (1984) for a general presentation of these tests). Let $\lambda \in \mathbb{R}^{s_{0}}$ be a Lagrange multiplier and let $\hat{\theta}_{n}^{c}$ be the LSE constrained by $H_{0}$ :

$$
\left(\hat{\theta}_{n}^{c}, \hat{\lambda}\right)=\arg \min _{\theta \in \Theta, \lambda \in \mathbb{R}^{s_{0}}} Q_{n}(\theta)-\lambda^{\prime}\left(R \theta-r_{0}\right) .
$$

For simplicity, consider the strong ARMA case. The asymptotic variance of the LSE can then be estimated either by

$$
\hat{\Omega}^{c}=\hat{\sigma}^{2 c}\left(\hat{J}_{n}^{c}\right)^{-1} \quad \text { or } \quad \hat{\Omega}^{* c}=\hat{\sigma}^{2 c}\left(\hat{J}_{n}^{* c}\right)^{-1}
$$

where

$$
\hat{J}_{n}^{c}=\frac{1}{n} \sum_{t=1}^{n} \frac{\partial \epsilon_{t}\left(\hat{\theta}_{n}^{c}\right)}{\partial \theta} \frac{\partial \epsilon_{t}\left(\hat{\theta}_{n}^{c}\right)}{\partial \theta^{\prime}}, \quad \hat{J}_{n}^{* c}=\frac{1}{n} \sum_{t=1}^{n} \epsilon_{t}\left(\hat{\theta}_{n}^{c}\right) \frac{\partial^{2} \epsilon_{t}\left(\hat{\theta}_{n}^{c}\right)}{\partial \theta \partial \theta^{\prime}}+\hat{J}_{n}^{c}
$$

and $\hat{\sigma}^{2 c}=n^{-1} \sum_{t=1}^{n} e_{t}^{2}\left(\hat{\theta}_{n}^{c}\right)$. This leads to two versions of the LM statistic

$$
\begin{aligned}
\mathbf{L M} & :=\frac{n}{\hat{\sigma}^{2 c}} \frac{\partial Q_{n}\left(\hat{\theta}_{n}^{c}\right)}{\partial \theta^{\prime}}\left(\hat{J}_{n}^{c}\right)^{-1} \frac{\partial Q_{n}\left(\hat{\theta}_{n}^{c}\right)}{\partial \theta}, \\
\mathbf{L M}^{*} & :=\frac{n}{\hat{\sigma}^{2 c}} \frac{\partial Q_{n}\left(\hat{\theta}_{n}^{c}\right)}{\partial \theta^{\prime}}\left(\hat{J}_{n}^{* c}\right)^{-1} \frac{\partial Q_{n}\left(\hat{\theta}_{n}^{c}\right)}{\partial \theta} .
\end{aligned}
$$

The two versions have the same asymptotic distribution under the null:

$$
\mathbf{L M} \stackrel{d}{\rightarrow} \chi_{s_{0}}^{2} \quad \text { and } \quad \mathbf{L M} \stackrel{d}{\rightarrow} \chi_{s_{0}}^{2} \quad \text { under } H_{0}
$$

but behaves differently under the alternative:

$$
\frac{\mathbf{L M}}{n} \rightarrow c:=D_{\theta_{0}^{c}}^{\prime}\left(\sigma^{2 c} J_{\theta_{0}^{c}}\right)^{-1} D_{\theta_{0}^{c}}, \quad \frac{\mathbf{L M}^{*}}{n} \rightarrow c^{*}:=D_{\theta_{0}^{c}}^{\prime}\left(\sigma^{2 c} J_{\theta_{0}^{c}}^{*}\right)^{-1} D_{\theta_{0}^{c}}
$$


under $H_{1}$ as $n \rightarrow \infty$, where

$$
D_{\theta}=E \epsilon_{t}(\theta) \frac{\partial \epsilon_{t}(\theta)}{\partial \theta}, \quad \sigma^{2 c}=E \epsilon_{1}^{2}\left(\theta_{0}^{c}\right)
$$

when $\hat{\theta}_{n}^{c} \rightarrow \theta_{0}^{c}$ a.s., where $\theta_{0}^{c}$ is such that $J_{\theta_{0}^{c}}$ and $J_{\theta_{0}^{c}}^{*}$ are positive-definite.

Note that $J_{\theta_{0}^{c}}$ is always positive-semidefinite, but this is not the case for $J_{\theta_{0}^{c}}^{*}$. When $J_{\theta_{0}^{c}}^{*}$ is not positive-definite, the $\mathbf{L M}^{*}$-test (i.e. the test of rejection region $\left\{\mathbf{L M}^{*} \geq \chi_{1}^{2}(1-\alpha)\right\}$, where $\alpha$ is the asymptotic level and $\chi_{k}^{2}(\alpha)$ denotes the $\alpha$-quantile of the chi-square distribution with $k$ degrees of freedom) may be inconsistent.

Thanks to the computation of Section 3, for any given alternative, we are able to determine which version is consistent and we are able to compute the Bahadur slopes. We now give a simple example in which hand-made computation of Bahadur's slopes is possible.

\subsubsection{Testing an $A R(1)$ against an $A R M A(1,1)$}

We now consider an $\operatorname{ARMA}(1,1)$ model and we test for an $\operatorname{AR}(1)$. We thus have $R=(0,1), r_{0}=0$ and $\theta_{0}^{c}=\left(a_{0}^{c}, 0\right)^{\prime}$ where

$$
a_{0}^{c}=\arg \min _{a} E\left(X_{t}-a X_{t-1}\right)^{2}=\rho(1),
$$

where $\rho(h)=\gamma(h) / \gamma(0)$ and $\gamma(h)=\operatorname{Cov}\left(X_{t}, X_{t-h}\right)$ denote respectively the autocorrelation and autocovariance of $\left(X_{t}\right)$ at lag $h$. Standard computations show that the constrained estimator satisfies

$$
\hat{\theta}_{n}^{c}=\left(\begin{array}{c}
\hat{a}^{c} \\
0
\end{array}\right), \quad \frac{\partial Q_{n}\left(\hat{\theta}_{n}^{c}\right)}{\partial \theta^{\prime}}=\left(\begin{array}{l}
0 \\
\hat{\lambda}
\end{array}\right)
$$

with

$$
\hat{a}^{c}=\frac{\sum_{t=2}^{n} X_{t} X_{t-1}}{\sum_{t=2}^{n} X_{t-1}^{2}}, \quad \hat{\lambda}=\hat{a}^{c} \frac{1}{n} \sum_{t=3}^{n} X_{t} X_{t-2}-\left(\hat{a}^{c}\right)^{2} \frac{1}{n} \sum_{t=3}^{n} X_{t-1} X_{t-2} .
$$


Other tedious computations show that

$$
\begin{aligned}
\sigma^{2 c} & =\left\{1+\left(a_{0}^{c}\right)^{2}\right\} \gamma(0)-2 a_{0}^{c} \gamma(1) \\
D_{\theta_{0}^{c}} & =-E\left(X_{t}-a_{0}^{c} X_{t-1}\right)\left(\begin{array}{c}
X_{t-1} \\
X_{t-1}-a_{0}^{c} X_{t-2}
\end{array}\right) \\
& =\left(\begin{array}{c}
0 \\
-\left(a_{0}^{c}\right)^{2} \gamma(1)+\left(a_{0}^{c}\right) \gamma(2)
\end{array}\right) \\
J_{\theta_{0}^{c}} & =\left(\begin{array}{cc}
\gamma(0) & \gamma(0)-a_{0}^{c} \gamma(1) \\
\gamma(0)-a_{0}^{c} \gamma(1) & \gamma(0)+\left(a_{0}^{c}\right)^{2} \gamma(0)-2 a_{0}^{c} \gamma(1)
\end{array}\right), \\
J_{\theta_{0}^{c}}^{*} & =J_{\theta_{0}^{c}}+E\left(X_{t}-a_{0}^{c} X_{t-1}\right)\left(\begin{array}{cc}
0 & X_{t-2} \\
X_{t-2} & 2\left(X_{t-2}-a_{0}^{c} X_{t-3}\right)
\end{array}\right) \\
& =J_{\theta_{0}^{c}}+\left(\begin{array}{cc}
0 & \gamma(2)-a_{0}^{c} \gamma(1) \\
\gamma(2)-a_{0}^{c} \gamma(1) & 2\left\{1+\left(a_{0}^{c}\right)^{2}\right\} \gamma(2)-2 a_{0}^{c}\{\gamma(1)+\gamma(3)\}
\end{array}\right) .
\end{aligned}
$$

The Bahadur slopes of the two versions of the LM tests are thus

$$
c=\frac{\left(a_{0}^{c} \gamma(1)-\gamma(2)\right)^{2} \gamma(0)}{\left\{\gamma^{2}(0)-\gamma^{2}(1)\right\} \sigma^{2 c}}
$$

and, under the assumption that the denominator is strictly positive,

$$
c^{*}=\frac{\left(a_{0}^{c} \gamma(1)-\gamma(2)\right)^{2} \gamma(0)}{\left\{\gamma^{2}(0)+2 \gamma(0) \gamma(2)-2 \frac{\gamma(0) \gamma(3)}{a_{0}^{c}}-\frac{\gamma^{2}(2)}{a_{0}^{c 2}}-4 \gamma^{2}(1)+4 \frac{\gamma(1) \gamma(2)}{a_{0}^{c}}\right\} \sigma^{2 c}} .
$$

In particular, it follows that, in the Bahadur sense, the $\mathbf{L M}^{*}$ version is more efficient than the LM one for MA(1) alternatives of the form $X_{t}=\epsilon_{t}+$ $b_{0} \epsilon_{t-1}$. Moreover, the asymptotic relative efficiency $c^{*} / c$ tends to infinity as $\left|b_{0}\right|$ approches 1 . For strong $\operatorname{ARMA}(1,1)$ alternatives of the form

$$
X_{t}-0.5 X_{t-1}=\epsilon_{t}+b_{0} \epsilon_{t-1}, \quad \epsilon_{t} \text { iid } \mathcal{N}(0,1) \text {, }
$$

tedious computations show that the $\mathbf{L M}^{*}$ version is inconsistent for $b_{0} \leq$ $-0.5807 \ldots$ but is more efficient than the $\mathbf{L M}$ version when $b_{0}>-0.5807 \ldots$ Figure 1 shows the percentage of Bahadar asymptotic relative efficiency (ARE) gain of $\mathbf{L M}^{*}$ with respect to $\mathbf{L M}$, as measured by $100\left(c^{*}-c\right) / c$, when $b_{0}$ varies from -0.45 to 0.55 . 


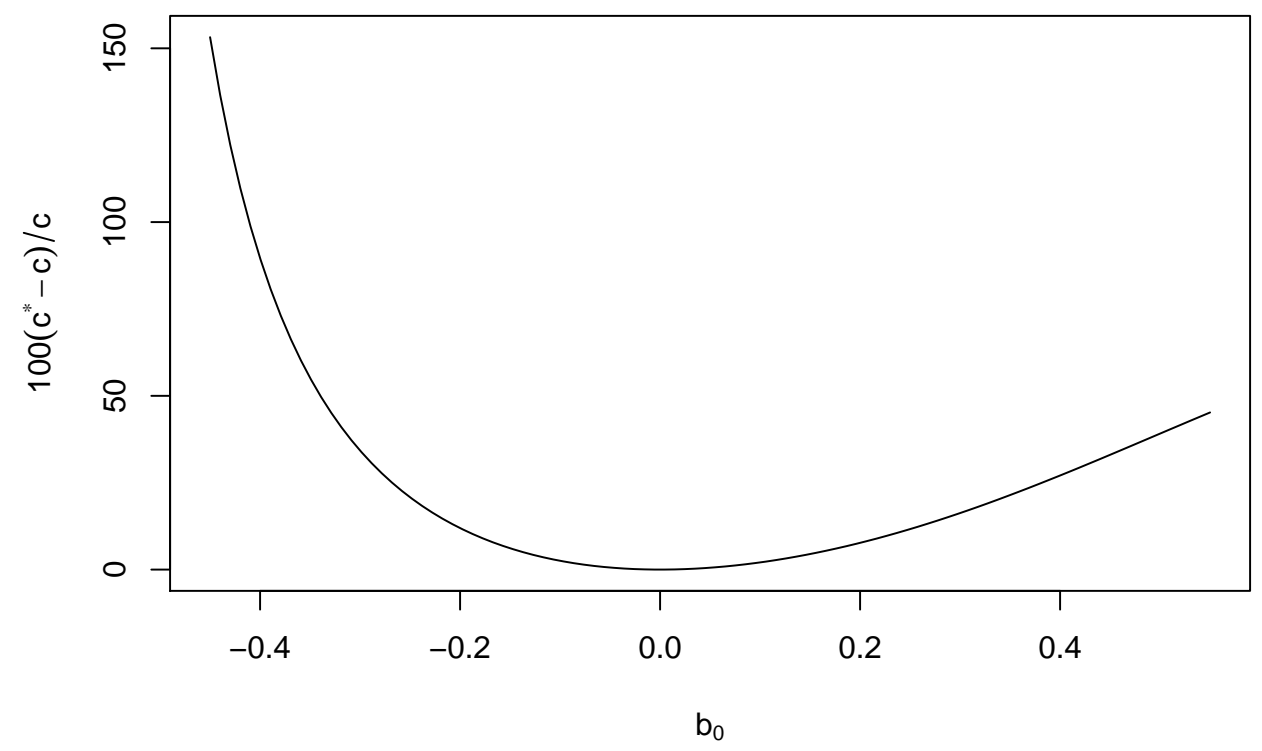

Figure 1: Relative improvement (in percentage) of the Bahadur slope of the $\mathbf{L M}^{*}$ test with respect to that of the $\mathbf{L M}$-test, when the null is $\operatorname{AR}(1)$ and the alternative is the $\operatorname{ARMA}(1,1)$ model $(18)$.

\subsubsection{Finite sample comparison of the two versions}

In order to determine whether the ARE computed in the previous section provide valuable insights on the actual behavior of the two tests, we simulated $N=1000$ independent trajectories of size $n=100, n=1000$ and $n=$ 10000 of the ARMA(1,1) models (18). Table 3 displays the averaged pvalues of the $\mathbf{L M}$ and $\mathbf{L M}^{*}$ tests of the null hypothesis of an $\mathrm{AR}(1)$, i.e. $H_{0}: b_{0}=0$. The two lines in bold correspond to the null hypothesis $H_{0}$. For the line $b_{0}=0$, the DGP is an $\mathrm{AR}(1)$ and the test statistics $\mathbf{L M}$ and $\mathbf{L M}^{*}$ are asymptotically $\chi_{1}^{2}$-distributed because

$$
J_{\theta_{0}^{c}}=J_{\theta_{0}^{c}}^{*}=\gamma(0)\left(\begin{array}{cc}
1 & 1-a_{0}^{2} \\
1-a_{0}^{2} & 1-a_{0}^{2}
\end{array}\right), \quad a_{0}=1 / 2
$$

is invertible and the arguments of Section 4.2.1 apply. For the line $b_{0}=-0.5$, the DGP is a white noise, which can also be written as an $\operatorname{ARMA}(1,1)$ with $a_{0}=b_{0}=0$, but the arguments of Section 4.2.1 do not apply because

$$
J_{\theta_{0}^{c}}=J_{\theta_{0}^{c}}^{*}=\gamma(0)\left(\begin{array}{ll}
1 & 1 \\
1 & 1
\end{array}\right)
$$

is singular. As expected, the average p-value of the $\mathbf{L M}$ and $\mathbf{L} \mathbf{M}^{*}$ tests are close to 0.5 when $b_{0}=0$. We also note the average p-value of the LM-test is 
close to 0.5 when $b_{0}=-0.5$, and is close to zero under the alternative when $n$ is large. In accordance with the theoretical results of the previous section, we also note that the $\mathbf{L} \mathbf{M}^{*}$-test is inconsistent for $b_{0}<-0.5$ because the $\mathbf{p}$ values do not tend to zero as $n$ increases. When $n$ is small and $b_{0}>0.5$, the p-values of the $\mathbf{L M}^{*}$-test are slightly smaller than those of the LM-test, but these p-values tend rapidly to zero as $n$ increases. For an easier comparison of the empirical behavior of the two tests, Table 4 reports the averages of the estimated Bahadur slopes $\mathbf{L M} / n$ and $\mathbf{L M}^{*} / n$. As expected from the asymptotic theory illustrated by Figure 1, the $\mathbf{L M}^{*}$ statistic is in average larger than the $\mathbf{L M}$ statistic for alternatives such that $b_{0}>-0.5$. Note also that the $\mathbf{L M}$ statistic is always positive, whereas negative values of $\mathbf{L} \mathbf{M}^{*}$ are observed, because $\hat{J}_{n}^{c}$ is semi-definite positive, whereas it is not the case for $\hat{J}_{n}^{* c}$.

To conclude this section, although the $\mathbf{L M}^{*}$ version may be asymptotically more efficient in Bahadur's sense than the LM version for particular alternatives, the $\mathbf{L M}$ version seems globally preferable because it is unbiased and consistent for a larger set of alternatives.

\subsection{Testing weak ARMA models for stock returns}

We now consider an application to the daily returns of 10 stock market indices (CAC, DAX, FTSE, HSI, Nikkei, NSE, SMI, SP500, SPTSX and SSE). The observations cover the period from the starting date of each index to July 26, 2010. In Financial Econometrics, the returns are often assumed to be martingale increments, and the squares of the returns have often secondorder moments close to those of an $\operatorname{ARMA}(1,1)$ (which is compatible with a $\operatorname{GARCH}(1,1)$ model for the returns).

We will test these hypotheses by fitting weak ARMA models on the returns and on their squares. In view of Section 4.1.2, let $\hat{\Omega}=J_{\hat{\theta}_{n}, n}^{-1} I_{\hat{\theta}_{n}, n} J_{\hat{\theta}_{n}, n}^{-1}$, $\hat{\Omega}_{s}=\hat{\sigma}^{2} J_{\hat{\theta}_{n}, n}^{-1}$ and $\hat{\Omega}_{s s}=J_{\hat{\theta}_{n}, n}^{-1} \hat{I}_{s s} J_{\hat{\theta}_{n}, n}^{-1}$. We will consider three versions of the Wald test of the null hypothesis defined in (17). Under the assumptions of Proposition 3.4 and the assumption that $I$ and $I_{s s}$ are invertible, the Wald statistics

$$
\begin{gathered}
\mathbf{W}_{S}=n\left(R_{0} \hat{\theta}_{n}-r_{0}\right)^{\prime}\left(R_{0} \hat{\Omega}_{s} R_{0}^{\prime}\right)^{-1}\left(R_{0} \hat{\theta}_{n}-r_{0}\right), \\
\mathbf{W}_{S S}=n\left(R_{0} \hat{\theta}_{n}-r_{0}\right)^{\prime}\left(R_{0} \hat{\Omega}_{s s} R_{0}^{\prime}\right)^{-1}\left(R_{0} \hat{\theta}_{n}-r_{0}\right), \\
\mathbf{W}_{W}=n\left(R_{0} \hat{\theta}_{n}-r_{0}\right)^{\prime}\left(R_{0} \hat{\Omega} R_{0}^{\prime}\right)^{-1}\left(R_{0} \hat{\theta}_{n}-r_{0}\right)
\end{gathered}
$$


Table 3: Averages of the p-values of $\mathbf{L M}$ and $\mathbf{L} \mathbf{M}^{*}$ for testing the null hypothesis of an $\operatorname{AR}(1)$, i.e. $H_{0}: b_{0}=0$, in the $\operatorname{ARMA}(1,1)$ model (18). The number of replications is $N=1000$.

\begin{tabular}{ccccccc}
\hline \hline & \multicolumn{2}{c}{$n=100$} & \multicolumn{2}{c}{$n=1,000$} & \multicolumn{2}{c}{$n=10,000$} \\
$b_{0}$ & $\mathbf{L M}$ & $\mathbf{L M}^{*}$ & $\mathbf{L M}$ & $\mathbf{L M}^{*}$ & $\mathbf{L M}$ & LM $^{*}$ \\
\hline-0.9 & 0.14185 & 0.82045 & 0.00000 & 0.98700 & 0.00000 & 1.00000 \\
-0.8 & 0.19998 & 0.78249 & 0.00028 & 0.94300 & 0.00000 & 1.00000 \\
-0.7 & 0.32798 & 0.77807 & 0.00917 & 0.81042 & 0.00000 & 0.98900 \\
-0.6 & 0.46288 & 0.81641 & 0.18710 & 0.67573 & 0.00004 & 0.61900 \\
$\mathbf{- 0 . 5}$ & $\mathbf{0 . 4 9 5 4 9}$ & $\mathbf{0 . 8 5 0 0 0}$ & $\mathbf{0 . 5 0 7 8 9}$ & $\mathbf{0 . 8 4 5 5 6}$ & $\mathbf{0 . 5 1 8 9 9}$ & $\mathbf{0 . 8 5 0 1 8}$ \\
-0.4 & 0.48500 & 0.77410 & 0.29030 & 0.48105 & 0.00252 & 0.01066 \\
-0.3 & 0.46271 & 0.65144 & 0.14221 & 0.13635 & 0.00000 & 0.00000 \\
-0.2 & 0.44848 & 0.50338 & 0.15298 & 0.14171 & 0.00000 & 0.00000 \\
-0.1 & 0.49491 & 0.51222 & 0.29239 & 0.28933 & 0.00368 & 0.00345 \\
$\mathbf{0 . 0}$ & $\mathbf{0 . 5 0 1 4 3}$ & $\mathbf{0 . 4 9 7 8 0}$ & $\mathbf{0 . 5 1 0 9 5}$ & $\mathbf{0 . 5 0 9 4 1}$ & $\mathbf{0 . 5 1 8 5 4}$ & $\mathbf{0 . 5 1 8 3 0}$ \\
0.1 & 0.44112 & 0.42938 & 0.17563 & 0.17287 & 0.00003 & 0.00003 \\
0.2 & 0.29159 & 0.27676 & 0.00483 & 0.00388 & 0.00000 & 0.00000 \\
0.3 & 0.14343 & 0.12986 & 0.00005 & 0.00000 & 0.00000 & 0.00000 \\
0.4 & 0.05982 & 0.04610 & 0.00000 & 0.00000 & 0.00000 & 0.00000 \\
0.5 & 0.01696 & 0.01194 & 0.00000 & 0.00000 & 0.00000 & 0.00000 \\
0.6 & 0.00752 & 0.00758 & 0.00000 & 0.00000 & 0.00000 & 0.00000 \\
0.7 & 0.00227 & 0.01223 & 0.00000 & 0.00000 & 0.00000 & 0.00000 \\
0.8 & 0.00156 & 0.01510 & 0.00000 & 0.00000 & 0.00000 & 0.00000 \\
0.9 & 0.00112 & 0.01109 & 0.00000 & 0.00000 & 0.00000 & 0.00000 \\
\hline \hline
\end{tabular}

asymptotically follow a $\chi_{s_{0}}^{2}$ distribution under $H_{0}$. At the asymptotic level $\alpha$, each Wald test consists in rejecting $H_{0}$ when its statistic is greater than $\chi_{s_{0}}^{2}(1-\alpha)$.

\subsubsection{Testing a white noise against an $A R(1)$}

In this section, we fit $\operatorname{AR}(1)$ models on each series of daily returns, and we apply the above-mentioned Wald tests for testing the hypothesis that the returns constitute a white noise. This testing problem can be trivially written under the form (17). Table 5 displays the $p$-values of the standard and 
Table 4: Estimated Bahadur slopes of the $\mathbf{L M}$ and $\mathbf{L M}^{*}$ tests, when the null hypothesis is an $\operatorname{AR}(1)$ and the alternative is the $\operatorname{ARMA}(1,1)$ model (18). The number of replications is $N=1000$.

\begin{tabular}{ccccccc}
\hline \hline & \multicolumn{2}{c}{$n=100$} & \multicolumn{2}{c}{$n=1,000$} & \multicolumn{2}{c}{$n=10,000$} \\
$b_{0}$ & LM & LM$^{*}$ & LM & LM $^{*}$ & LM & LM $^{*}$ \\
\hline-0.9 & 0.04712 & 4.17203 & 0.03717 & -0.05981 & 0.03631 & -0.04923 \\
-0.8 & 0.03654 & -0.13111 & 0.02654 & 5.93785 & 0.02589 & -0.04407 \\
-0.7 & 0.02320 & 0.98988 & 0.01376 & -0.03605 & 0.01269 & -0.04327 \\
-0.6 & 0.01280 & -0.00635 & 0.00404 & 0.01628 & 0.00313 & 0.00297 \\
$\mathbf{- 0 . 5}$ & $\mathbf{0 . 0 1 0 3 5}$ & $\mathbf{- 0 . 0 0 2 1 1}$ & $\mathbf{0 . 0 0 1 0 2}$ & $\mathbf{0 . 0 0 0 2 0}$ & $\mathbf{0 . 0 0 0 1 0}$ & $\mathbf{0 . 0 0 0 0 1}$ \\
-0.4 & 0.01050 & -0.01204 & 0.00269 & 0.00062 & 0.00186 & 0.00449 \\
-0.3 & 0.01282 & 0.00389 & 0.00482 & 0.00794 & 0.00425 & 0.00591 \\
-0.2 & 0.01240 & 0.01819 & 0.00487 & 0.00600 & 0.00417 & 0.00471 \\
-0.1 & 0.01060 & 0.00009 & 0.00271 & 0.00287 & 0.00180 & 0.00185 \\
$\mathbf{0 . 0}$ & $\mathbf{0 . 0 1 0 3 6}$ & $\mathbf{0 . 0 0 7 2 1}$ & $\mathbf{0 . 0 0 0 9 2}$ & $\mathbf{0 . 0 0 0 9 5}$ & $\mathbf{0 . 0 0 0 0 8}$ & $\mathbf{0 . 0 0 0 0 8}$ \\
0.1 & 0.01393 & 0.01720 & 0.00422 & 0.00438 & 0.00335 & 0.00343 \\
0.2 & 0.02607 & 0.03886 & 0.01600 & 0.01750 & 0.01510 & 0.01627 \\
0.3 & 0.04626 & 0.05910 & 0.03781 & 0.04417 & 0.03660 & 0.04254 \\
0.4 & 0.07466 & 0.22160 & 0.06637 & 0.08452 & 0.06550 & 0.08327 \\
0.5 & 0.10383 & 0.17138 & 0.09796 & 0.13724 & 0.09764 & 0.13601 \\
0.6 & 0.13199 & 0.28353 & 0.12860 & 0.19518 & 0.12850 & 0.19402 \\
0.7 & 0.15988 & 0.28823 & 0.15401 & 0.25127 & 0.15339 & 0.24780 \\
0.8 & 0.17431 & 0.34168 & 0.16972 & 0.29062 & 0.17080 & 0.28910 \\
0.9 & 0.18064 & 0.33781 & 0.17949 & 0.31841 & 0.18062 & 0.31362 \\
\hline \hline
\end{tabular}

modified Wald tests. For the NSE, SMI, SP500 and SPTSX series, the white noise hypothesis is rejected by the $\mathbf{W}_{S}$ test at the nominal level $\alpha=5 \%$. This is not surprising because the $\mathbf{W}_{S}$ test required the iid assumption and, in particular in view of the so-called volatility clustering, it is well known that the strong white noise model is not adequate for these series. By contrast, the white noise hypothesis is not rejected by the modified tests based on $\mathbf{W}_{S S}$ and $\mathbf{W}_{W}$. To summary, the outputs of Table 5 are in accordance with the common belief that these series are not strong white noises, but could be weak white noises (or even martingale increments). 
We now turn to the dynamics of the squared returns.

Table 5: For standard and modified versions of Wald tests, $p$-values of the null hypothesis that the returns are white noises. The $p$-values which are less than $\alpha=5 \%$ are displayed in bold.

\begin{tabular}{lrrrr}
\hline \hline Returns & Length $n$ & $\mathbf{W}_{S}$ & $\mathbf{W}_{S S}$ & $\mathbf{W}_{W}$ \\
CAC & 5154 & 0.386 & 0.570 & 0.486 \\
DAX & 4966 & 0.343 & 0.521 & 0.349 \\
FTSE & 6647 & 0.705 & 0.857 & 0.760 \\
HSI & 5849 & 0.144 & 0.631 & 0.356 \\
Nikkei & 6530 & 0.057 & 0.328 & 0.159 \\
NSE & 1990 & $\mathbf{0 . 0 3 8}$ & 0.250 & 0.082 \\
SMI & 4963 & $\mathbf{0 . 0 3 5}$ & 0.264 & 0.060 \\
SP500 & 15237 & $\mathbf{0 . 0 0 0}$ & 0.073 & 0.019 \\
SPTSX & 2665 & $\mathbf{0 . 0 4 2}$ & 0.321 & 0.105 \\
SSE & 2716 & 0.707 & 0.781 & 0.758 \\
\hline \hline
\end{tabular}

\subsubsection{Testing the ARMA(1,1) model for the squared returns}

We fitted $\operatorname{ARMA}(p, q)$ models with $p=1$ and $q>1$, or $q=1$ and $p>1$, on the squares of the previous daily returns, and we applied Wald tests for testing the null hypothesis of an $\operatorname{ARMA}(1,1)$ model. The $p$-values of the standard and modified Wald tests are displayed in Table 6. The standard Wald test frequently rejects the $\operatorname{ARMA}(1,1)$ model. The validity of this test is however questionable, because the assumption of iid linear innovations is not very plausible, as well for the squared returns than for the returns themselves (as was discussed in the previous section). If the returns are assumed to follow a $\operatorname{GARCH}(1,1)$, which is one of the most widely used model for such series, then the squared returns follow a semi-strong $\operatorname{ARMA}(1,1)$, and higher-order powers follow ARMA models which are only weak (see). The tests based on the statistics $\mathbf{W}_{S S}$ and $\mathbf{W}_{W}$ thus appear as more reliable, a priori. These tests also frequently reject the ARMA $(1,1)$ model in favor of more complex models. This leads us to reconsider the common belief that the GARCH $(1,1)$ model is sufficient to capture the dynamics of most financial returns, and that higher-order models would be unnecessarily complicated. Francq and Zakoïan (2009a) drew the same conclusion from parametric tests 
on GARCH models. The advantage of the present study is that it leads to reconsider not only the $\mathrm{GARCH}(1,1)$ model, but also any parametric model leading to a weak $\operatorname{ARMA}(1,1)$ for the squares.

\section{Conclusion}

The asymptotic variance of the LSE of ARMA models depend on information matrices $I$ and $J$ computed at the true value of the parameter $\theta_{0}$. It is sometime necessary to evaluate these matrices at some point $\theta \neq \theta_{0}$. In the case of strong ARMA models, $I_{\theta}$ and $J_{\theta}$ depend only on $\theta_{0}$ and on the moments $\sigma^{2}=E \epsilon_{t}^{2}$ and $\mu_{4}=E \epsilon_{t}^{4}$ of the iid noise $\epsilon_{t}$. In the much more general case of weak ARMA models, $I_{\theta}$ also depends on the autocovariances of the weak white noise $\epsilon_{t}^{2}$.

We proposed here algorithms for the exact computation of $I_{\theta}$ and $J_{\theta}$ from the model, and for the estimation of these matrices from the data. It is possible to define estimators of the information matrices which are consistent in the general weak case, or in the more restrictive semi-strong case, or only in the strong case. Simulations experiments confirmed the domain of validity of the different estimators, and also that an efficiency loss is the price to pay for having more robust estimators. As an illustration of the interest of considering $I_{\theta}$ and $J_{\theta}$ at $\theta \neq \theta_{0}$, we computed and compared Bahadur's slopes of two versions of the Lagrange-Multiplier test for testing general linear restriction on $\theta_{0}$ in the strong ARMA case. The two versions are based on two estimators $\hat{J}_{n}^{c}$ and $\hat{J}_{n}^{* c}$ of $J$ under the null. The standard estimator of $J$ is $\hat{J}_{n}^{c}$, whereas $\hat{J}_{n}^{* c}$ contains an extra term which is asymptotically negligible under the null but may have importance under the alternative. We showed, analytically and also by means of simulations, that the version based on $\hat{J}_{n}^{* c}$ may be asymptotically much more efficient than the standard version, but is consistent for a narrower set of alternatives. Applying different Wald tests based on different estimators of the information matrices, and applying them for testing weak ARMA specifications on daily stock returns and on their squares, we reconsidered models such as the popular $\operatorname{GARCH}(1,1)$ for which the squares follow a weak $\operatorname{ARMA}(1,1)$. 
Table 6: As Table 5, but for the null hypothesis that the squared returns follow an $\operatorname{ARMA}(1,1)$ model.

\begin{tabular}{|c|c|c|c|c|}
\hline Alternative & Returns & $\mathbf{W}_{S}$ & $\mathbf{W}_{S S}$ & $\mathbf{W}_{W}$ \\
\hline \multirow{10}{*}{$\operatorname{ARMA}(2,1)$} & $\overline{\mathrm{CAC}}$ & 0.000 & 0.228 & 0.167 \\
\hline & DAX & 0.000 & 0.013 & 0.000 \\
\hline & FTSE & 0.767 & 0.949 & 0.962 \\
\hline & HSI & 0.000 & 0.351 & 0.000 \\
\hline & Nikkei & 0.483 & 0.922 & 0.940 \\
\hline & NSE & 0.027 & 0.485 & 0.570 \\
\hline & SMI & 0.589 & 0.886 & 0.803 \\
\hline & SP500 & 0.014 & 0.641 & 0.503 \\
\hline & SPTSX & 0.009 & 0.472 & 0.443 \\
\hline & SSE & 0.042 & 0.281 & 0.139 \\
\hline \multirow{10}{*}{$\operatorname{ARMA}(1,2)$} & $\overline{\mathrm{CAC}}$ & 0.000 & 0.288 & 0.064 \\
\hline & DAX & 0.000 & 0.060 & 0.013 \\
\hline & FTSE & 0.828 & 0.957 & 0.941 \\
\hline & HSI & 0.000 & 0.005 & 0.000 \\
\hline & Nikkei & 0.594 & 0.936 & 0.938 \\
\hline & NSE & 0.053 & 0.305 & 0.191 \\
\hline & SMI & 0.668 & 0.897 & 0.863 \\
\hline & SP500 & 0.069 & 0.737 & 0.793 \\
\hline & SPTSX & 0.001 & 0.312 & 0.571 \\
\hline & SSE & 0.040 & 0.067 & 0.025 \\
\hline \multirow{10}{*}{$\operatorname{ARMA}(1,3)$} & $\overline{\mathrm{CAC}}$ & 0.003 & 0.617 & 0.228 \\
\hline & DAX & 0.000 & 0.012 & 0.000 \\
\hline & FTSE & 0.000 & 0.396 & 0.181 \\
\hline & HSI & 0.000 & 0.000 & 0.000 \\
\hline & Nikkei & 0.000 & 0.419 & 0.000 \\
\hline & NSE & 0.286 & 0.742 & 0.621 \\
\hline & SMI & 0.000 & 0.328 & 0.041 \\
\hline & SP500 & 0.000 & 0.000 & 0.000 \\
\hline & SPTSX & 0.000 & 0.001 & 0.000 \\
\hline & SSE & 0.006 & 0.022 & 0.001 \\
\hline
\end{tabular}




\section{A. Proofs}

Proof of Proposition 3.3. The first result follows from (7) and

$$
I_{\theta}(i, k)=\sum_{h=-\infty}^{+\infty} \sum_{h_{1}, h_{2}, h_{3}, h_{4}=0}^{+\infty} c_{h_{1}} c_{h_{2}}^{a} c_{h_{3}} c_{h_{4}}^{a} \operatorname{Cov}\left(\epsilon_{t-h_{1}} \epsilon_{t-h_{2}-i}, \epsilon_{t-h-h_{3}} \epsilon_{t-h-h_{4}-k}\right) .
$$

The other results follow similarly.

Proof of Proposition 3.3. Let $\tilde{c}_{h}=c_{h}$ for $0 \leq h \leq M$ and $\tilde{c}_{h}=0$ for $h>M$. Similarly, we defined $\tilde{c}_{h}^{a}$ and $\tilde{c}_{h}^{b}$. For $1 \leq i \leq k \leq p$, we have

$$
\begin{aligned}
& I_{\theta}(i, k)-I_{\theta}^{M}(i, k)=\sum_{h_{1}, h_{2}, h_{3}, h_{4}=0}^{\infty}\left\{\left(c_{h_{1}}-\tilde{c}_{h_{1}}\right) c_{h_{2}}^{a} c_{h_{3}} c_{h_{4}}^{a}+\tilde{c}_{h_{1}}\left(c_{h_{2}}^{a}-\tilde{c}_{h_{2}}^{a}\right) c_{h_{3}} c_{h_{4}}^{a}\right. \\
& \left.+\tilde{c}_{h_{1}} \tilde{c}_{h_{2}}^{a}\left(c_{h_{3}}-\tilde{c}_{h_{3}}\right) c_{h_{4}}^{a}+\tilde{c}_{h_{1}} \tilde{c}_{h_{2}}^{a} \tilde{c}_{h_{3}}\left(c_{h_{4}}^{a}-\tilde{c}_{h_{4}}^{a}\right)\right\} \Gamma\left(h_{2}+i-h_{1}, h_{4}+k-h_{3}\right) .
\end{aligned}
$$

Note that if

$$
\max _{i=1, \ldots, k}\left|\rho_{i}\right| \leq \bar{\rho}<1
$$

then for all $|z| \leq 1$,

$$
\frac{1}{\prod_{i=1}^{k}\left(1-\rho_{i} z\right)}=\sum_{h=0}^{\infty} d_{h} z^{h}, \quad \text { with } \quad\left|d_{h}\right| \leq(h+1)^{k-1} \bar{\rho}^{h} .
$$

Note also that

$$
\phi(z) \psi_{0}(z)=\left(1-\sum_{i=1}^{p} a_{i} z^{i}\right)\left(1+\sum_{j=1}^{q} b_{0 j} z^{j}\right)=\sum_{\ell=0}^{p+q} \pi_{\ell} z^{\ell},
$$

where, with the convention $a_{0}=-1, b_{00}=1$ and $a_{i}=0$ for $i<0$,

$$
\left|\pi_{\ell}\right|=\left|\sum_{j=0}^{q} b_{0 j} a_{\ell-j}\right| \leq \bar{\pi}
$$

We thus have

$$
\max \left\{\left|c_{h}\right|,\left|c_{h}^{a}\right|,\left|c_{h}^{b}\right|\right\} \leq(p+q+1) \bar{\pi}(h+1)^{k_{0}} \bar{\rho}^{h} \leq K_{0} \bar{\rho}^{h / 2}
$$

with

$$
k_{0}=p+2 q-1, \quad K_{0}=(p+q+1) \bar{\pi}\left(\frac{-2 k_{0}}{\log \bar{\rho}}\right)^{k_{0}} \bar{\rho}^{-0.5-k_{0} / \log \bar{\rho}} .
$$


We then obtain

$$
\left|I_{\theta}(i, k)-I_{\theta}^{M}(i, k)\right| \leq 4 \bar{\Gamma}\left(\frac{K}{1-\bar{\rho}^{1 / 2}}\right)^{4} \bar{\rho}^{(M+1) / 2}
$$

and the result follows.

In the following proofs, $K$ and $\rho$ denote generic constant such as $K>0$ and $\rho \in(0,1)$, whose exact values are unimportant.

Proof of Proposition 3.4. Note that because the roots $\psi$ and $\phi_{0}$ are outside the unit circle,

$$
\max \left\{\left|c_{h}^{a}\right|,\left|c_{h}^{b}\right|,\left|c_{h}^{a b}\right|,\left|c_{h}^{b b}\right|\right\} \leq K \rho^{h} .
$$

Because $\Theta^{*}$ is compact, this inequality holds uniformly in $\theta \in \Theta^{*}$. Note that this entails the existence of $J_{\theta^{*}}$ and $J_{\theta^{*}}^{*}$. The ergodic theorem then shows that

$$
J_{\theta^{*}, n} \rightarrow J_{\theta^{*}} \text { and } J_{\theta^{*}, n}^{*} \rightarrow J_{\theta^{*}}^{*} \text { a.s. }
$$

By the previous arguments, for all $i, i=1, \ldots, p+q$, we have

$$
E \sup _{\theta \in \Theta^{*}}\left\|\frac{\partial}{\partial \theta} \frac{\partial \epsilon_{t}(\theta)}{\partial \theta_{i}} \frac{\partial \epsilon_{t}(\theta)}{\partial \theta_{j}}\right\|<\infty
$$

A Taylor expansion yields

$$
J_{\theta_{n}^{*}, n}(i, j)=J_{\theta^{*}, n}(i, j)+\left(\theta_{n}^{*}-\theta^{*}\right)^{\prime} \frac{1}{n} \sum_{t=1}^{n} \frac{\partial}{\partial \theta}\left\{\frac{\partial \epsilon_{t}(\theta)}{\partial \theta_{i}} \frac{\partial \epsilon_{t}(\theta)}{\partial \theta_{j}}\right\}\left(\theta^{* *}\right)
$$

for some $\theta^{* *}$ between $\theta_{n}^{*}$ and $\theta^{*}$. The consistency of $J_{\theta_{n}^{*}, n}$ follows from (20), (21) and the convergence of $\theta_{n}^{*}$ to $\theta^{*}$. The consistency of $J_{\theta_{n}^{*}, n}^{*}$ is shown similarly.

\section{References}

Andrews, D.W.K. Heteroskedasticity and autocorrelation consistent covariance matrix estimation. Econometrica 59:817-858, 1991.

Berk, K.N. Consistent Autoregressive Spectral Estimates. Ann. Stat. 2:489502, 1974. 
Box, G.E.P. and Jenkins, G.M. Time Series Analysis: Forecasting and Control, 2nd edition. Holden-Day, San Francisco, 1976.

Bradley, R.C. Basic properties of strong mixing conditions. A survey and some open questions. Probability Surveys 2:107-144, 2005.

Brockwell, P.J. and Davis, R.A. Time series: theory and methods. Springer Verlag, New York, 1991.

Durbin, J. Efficient Estimation of Parameters in Moving Average Models. Biometrika 46:306-316, 1959.

den Haan, W.J. and Levin, A. A Practitioner's Guide to Robust Covariance Matrix Estimation. In Handbook of Statistics 15, Rao, C.R. and G.S. Maddala (eds), 291-341, 1997.

Engle, R.F. Wald, likelihood ratio, and Lagrange multiplier tests in econometrics. Handbook of Econometrics 2, Z. Griliches and M.D. Intriligator (eds), Amsterdam: North-Holland, 775-826, 1984.

Francq, C., Roy, R. and Zakoïan, J-M. Diagnostic checking in ARMA Models with Uncorrelated Errors. Journal of the American Statistical Association 100:532-544, 2005.

Francq, C. and Zakoïan, J-M. Estimating linear representations of nonlinear processes. Journal of Statistical Planning and Inference 68:145-165, 1998.

Francq, C. and Zakoïan, J-M. Covariance matrix estimation for estimators of mixing weak ARMA models. Journal of Statistical Planning and Inference 83:369-394, 2000 .

Francq, C. and Zakoïan, J-M. Recent results for linear time series models with non independent innovations. In Statistical Modeling and Analysis for Complex Data Problems, Chap. 12 (eds P. DuChesne and B. RÉmilLARD). New York: Springer Verlag, 241-265, 2005.

Francq, C. and Zakoïan, J-M. HAC estimation and strong linearity testing in weak ARMA models. Journal of Multivariate Analysis 98:114-144, 2007.

Francq, C. and Zakoïan, J-M. Testing the nullity of GARCH coefficients: correction of the standard tests and relative efficiency comparisons. Journal of the American Statistical Association 104:313-324, 2009a. 
Francq, C. and Zakoïan, J-M. Bartlett's formula for a general class of non linear processes. Journal of Time Series Analysis 30:449-465, 2009b.

Francq, C. and Zakoïan, J-M. GARCH Models: Structure, Statistical Inference and Financial Applications. Wiley, 2010.

Godolphin, E.J. and Bane, S.R. On the evaluation of the information matrix for multiplicative seasonal time series. Journal of Time Series Analysis 27: 167-190, 2006.

Klein, A. and Mélard, G. Fisher's information matrix for seasonal moving average models. Journal of Time Series Analysis 11: 231-237, 1990.

Klein, A. and Mélard, G. An algorithm for computing the asymptotic Fisher information matrix for seasonal SISO models. Journal of Time Series Analysis 25: 627-648, 2004.

McKenzie, E. Discrete variate time series. In Handbook of Statistics, Rao, C.R. and D. Shanbhag (eds), Elsevier Science, Amsterdam, 573-606, 2003.

McLeod, A.I. On the distribution of residual autocorrelations in Box-Jenkins models. Journal of the Royal Statistical Society B 40:296-302, 1978.

McLeod, A.I. Duality and other properties of multiplicative seasonal autoregressive-moving average models. Biometrika 71:207-211, 1984.

Newey, W.K. and West, K.D. A simple, positive semi-definite, heteroskedasticity and autocorrelation consistent covariance matrix. Econometrica 55:703-708, 1987.

Romano, J.L. and Thombs, L.A. Inference for autocorrelations under weak assumptions. Journal of the American Statistical Association 91:590-600, 1996.

Siddiqui, M.M. On the inversion of the sample covariance matrix in a stationary autogressive process. Annals of Mathematical Statistics 29:585-588, 1958.

Whittle, P. Estimation and information in stationary time series. Arkiv för Matematik 2: 423-434, 1953. 
Whittle, P. On the fitting of multivariate autoregressions and the approximate canonical factorization of a special density matrix. Biometrika 40:129-134, 1963. 


\section{Computing and estimating information matrices of weak ARMA models: a complementary result which is not submitted for publication}

\section{A. Asymptotic properties of the spectral density estimator of the long-run variance $\boldsymbol{I}_{\theta^{*}}$}

Theorem A.1. Let the assumptions of Lemma 2.1 be satisfied. Assume that the process $\left(\nabla_{t}\right)$ defined by (12) admits the $A R(\infty)$ representation (13), where $\left\|A_{i}\right\|=o\left(i^{-2}\right)$ as $i \rightarrow \infty$, the roots of $\operatorname{det}(\mathcal{A}(z))=0$ are outside the unit disk, and $\Sigma_{u}$ is non-singular. Assume moreover that $E\left|\epsilon_{t}\right|^{8+4 \nu}<\infty$ and $\sum_{k=0}^{\infty}\left\{\alpha_{\epsilon}(k)\right\}^{\nu /(2+\nu)}<\infty$ for some $\nu>0$. Then, if $\theta_{n}^{*} \rightarrow \theta^{*}$ almost surely,

$$
I_{\theta_{n}^{*}, n}=\hat{\mathcal{A}}_{r}^{-1}(1) \hat{\Sigma}_{u_{r}} \hat{\mathcal{A}}_{r}^{\prime-1}(1) \rightarrow I_{\theta^{*}}
$$

in probability when $r=r(n) \rightarrow \infty$ and $r^{3} / n \rightarrow 0$ as $n \rightarrow \infty$.

The proof of Theorem A.1 is based on a series of lemmas. We use the multiplicative matrix norm defined by: $\|A\|=\sup _{\|x\| \leq 1}\|A x\|=\varrho^{1 / 2}\left(A^{\prime} A\right)$, where $A$ is a $d_{1} \times d_{2}$ matrix, $\|x\|$ is the Euclidean norm of the vector $x \in \mathbb{R}^{d_{2}}$, and $\varrho(\cdot)$ denotes the spectral radius. This norm satisfies

$$
\|A\|^{2} \leq \sum_{i, j} a_{i, j}^{2}
$$

with obvious notations. This choice of the norm is crucial for the following lemma to hold (with e.g. the Euclidean norm, this result is not valid). Let

$$
\Sigma_{\nabla, \underline{\nabla}_{r}}=E \nabla_{t} \underline{\nabla}_{r, t}^{\prime}, \quad \Sigma_{\nabla}=E \nabla_{t} \nabla_{t}^{\prime}, \quad \Sigma_{\underline{\nabla}_{r}}=E \underline{\nabla}_{r, t} \underline{\nabla}_{r, t}^{\prime} .
$$

In the sequel, $K$ and $\rho$ denote generic constant such as $K>0$ and $\rho \in(0,1)$, whose exact values are unimportant.

Lemma 1. Under the assumptions of Theorem A.1,

$$
\sup _{r \geq 1} \max \left\{\left\|\Sigma_{\nabla, \underline{\Xi}_{r}}\right\|,\left\|\Sigma_{\underline{\Xi}_{r}}\right\|,\left\|\Sigma_{\underline{\Xi}_{r}}^{-1}\right\|\right\} \leq \infty .
$$


Proof. We readily have

$$
\left\|\Sigma_{\underline{\nabla}_{r}} x\right\| \leq\left\|\Sigma_{\underline{\nabla}_{r+1}}\left(x^{\prime}, 0_{p+q}^{\prime}\right)^{\prime}\right\| \quad \text { and } \quad\left\|\Sigma_{\nabla, \underline{\nabla}_{r}} x\right\| \leq\left\|\Sigma_{\underline{\nabla}_{r+1}}\left(0_{p+q}^{\prime}, x^{\prime}\right)^{\prime}\right\|
$$

for any $x \in \mathbb{R}^{(p+q) r}$. Therefore

$$
0<\left\|\operatorname{Var}\left(\nabla_{t}\right)\right\|=\left\|\Sigma_{\underline{\nabla}_{1}}\right\| \leq\left\|\Sigma_{\underline{\nabla}_{2}}\right\| \leq \cdots
$$

and

$$
\left\|\Sigma_{\nabla, \underline{\nabla}_{r}}\right\| \leq\left\|\Sigma_{\underline{\nabla}_{r+1}}\right\| .
$$

Let $f(\lambda)$ be the spectral density of $\nabla_{t}$. Because the autocovariance function of $\nabla_{t}$ is absolutely summable, $\|f(\lambda)\|$ is bounded by a finite constant $K$, say. Denoting by $\delta=\left(\delta_{1}^{\prime}, \ldots, \delta_{r}^{\prime}\right)^{\prime}$ an eigenvector of $\Sigma_{\underline{\nabla}_{r}}$ associated with its largest eigenvalue, such that $\|\delta\|=1$ and $\delta_{i} \in \mathbb{R}^{p+q}$ for $\bar{i}=1, \ldots, r$, we have

$$
\begin{aligned}
\left\|\Sigma_{\underline{\nabla}_{r}}\right\| & =\varrho^{1 / 2}\left(\Sigma_{\underline{\nabla}_{r}}^{2}\right)=\varrho\left(\Sigma_{\underline{\nabla}_{r}}\right)=\delta^{\prime} \Sigma_{\underline{\nabla}_{r}} \delta \\
& =\sum_{j, k=1}^{r} \delta_{j}^{\prime} \int_{-\pi}^{\pi} e^{i(k-j) \lambda} f(\lambda) d(\lambda) \delta_{k} \leq 2 \pi K .
\end{aligned}
$$

By similar arguments, the smallest eigenvalue of $\Sigma_{\underline{\underline{V}}_{r}}$ is greater than a positive constant independent of $r$. Using the fact that $\left\|\underline{\Sigma}_{\underline{\nabla}_{r}}^{-1}\right\|$ is equal to the inverse of the smallest eigenvalue of $\Sigma_{\underline{\underline{Z}}_{r}}$, the proof is completed.

Denote by $\nabla_{t}(i)$ the $i$-th element of $\nabla_{t}$.

Lemma 2. Under the assumptions of Theorem A.1, there exits a finite constant $K_{1}$ such that for $m_{1}, m_{2}=1, \ldots, p+q$

$$
\sup _{s \in \mathbb{Z}} \sum_{h=-\infty}^{\infty}\left|\operatorname{Cov}\left\{\nabla_{1}\left(m_{1}\right) \nabla_{1+s}\left(m_{2}\right), \nabla_{1+h}\left(m_{1}\right) \nabla_{1+s+h}\left(m_{2}\right)\right\}\right|<K_{1} .
$$

Proof. Without loss of generality, we can take the supremum over the integers $s>0$, and write the proof in the case $m_{1}=m_{2}=m$. In view of (19), we have

$$
\begin{aligned}
& \sum_{h=-\infty}^{\infty}\left|\operatorname{Cov}\left\{\nabla_{1}(m) \nabla_{1+s}(m), \nabla_{1+h}(m) \nabla_{1+s+h}(m)\right\}\right| \\
\leq & K^{8} \sum_{h_{1}, \ldots, h_{8}=0}^{\infty} \rho^{\sum_{i=1}^{8} h_{i}} \sum_{h=-\infty}^{\infty}\left|\operatorname{Cov}\left(Y_{1, h_{1}, h_{2}} Y_{1+s, h_{3}, h_{4}}, Y_{1+h, h_{5}, h_{6}} Y_{1+s+h, h_{7}, h_{8}}\right)\right|
\end{aligned}
$$


where

$$
Y_{t, h_{1}, h_{2}}=\epsilon_{t-h_{1}} \epsilon_{t-h_{2}-m}-E \epsilon_{t-h_{1}} \epsilon_{t-h_{2}-m}
$$

A slight extension of Corollary A.3 in Francq and Zakoian (2010) concludes.

Let $\hat{\Sigma}_{\underline{\nabla}_{r}}, \hat{\Sigma}_{\nabla}$ and $\hat{\Sigma}_{\nabla, \underline{\nabla}_{r}}$ be the matrices obtained by replacing $\hat{\nabla}_{t}$ by $\nabla_{t}$ in $\hat{\Sigma}_{\hat{\underline{\Sigma}}_{r}}, \hat{\Sigma}_{\hat{\nabla}}$ and $\hat{\Sigma}_{\hat{\nabla}, \hat{\underline{\Sigma}}_{r}}$.

Lemma 3. Under the assumptions of Theorem A.1, $\sqrt{r}\left\|\hat{\Sigma}_{\underline{\nabla}_{r}}-\Sigma_{\underline{\nabla}_{r}}\right\|$, $\sqrt{r}\left\|\hat{\Sigma}_{\nabla}-\Sigma_{\nabla}\right\|$, and $\sqrt{r}\left\|\hat{\Sigma}_{\nabla, \underline{\nabla}_{r}}-\Sigma_{\nabla, \underline{\nabla}_{r}}\right\|$ tend to zero in probability as $n \rightarrow \infty$ when $r=o\left(n^{1 / 3}\right)$.

Proof. For $1 \leq m_{1}, m_{2} \leq p+q$ and $1 \leq r_{1}, r_{2} \leq r$, the element of the $\left\{\left(r_{1}-1\right)(p+q)+m_{1}\right\}$-th row and $\left\{\left(r_{2}-1\right)(p+q)+m_{2}\right\}$-th column of $\hat{\Sigma}_{\underline{\nabla}_{r}}$ is of the form $n^{-1} \sum_{t=1}^{n} Z_{t}$ where $Z_{t}=\nabla_{t-r_{1}}\left(m_{1}\right) \nabla_{t-r_{2}}\left(m_{2}\right)$. By stationarity of $\left(Z_{t}\right)$, we have

$$
\operatorname{Var}\left(\frac{1}{n} \sum_{t=1}^{n} Z_{t}\right)=\frac{1}{n^{2}} \sum_{h=-n+1}^{n-1}(n-|h|) \operatorname{Cov}\left(Z_{t}, Z_{t-h}\right) \leq \frac{K_{1}}{n},
$$

where, by Lemma $2, K_{1}$ is a constant independent of $r_{1}, r_{2}, m_{1}, m_{2}$ and $r, n$.

In view of (22) and (23) we have

$$
\begin{aligned}
& E\left\{r\left\|\hat{\Sigma}_{\nabla}-\Sigma_{\nabla}\right\|^{2}\right\} \leq E\left\{r\left\|\hat{\Sigma}_{\nabla, \underline{\nabla}_{r}}-\Sigma_{\nabla, \underline{\nabla}_{r}}\right\|^{2}\right\} \\
& \leq E\left\{r\left\|\hat{\Sigma}_{\underline{\nabla}_{r}}-\Sigma_{\underline{\nabla}_{r}}\right\|^{2}\right\} \leq \frac{K_{1}(p+q)^{2} r^{3}}{n}=o(1)
\end{aligned}
$$

as $n \rightarrow \infty$ when $r=o\left(n^{1 / 3}\right)$. The result follows. $\hat{\nabla}_{t}$.

We now show that the previous lemma applies when $\nabla_{t}$ is replaced by

Lemma 4. Under the assumptions of Theorem A.1, $\sqrt{r}\left\|\hat{\Sigma}_{\hat{\underline{\nabla}}_{r}}-\Sigma_{\underline{\nabla}_{r}}\right\|$, $\sqrt{r}\left\|\hat{\Sigma}_{\hat{\nabla}}-\Sigma_{\nabla}\right\|$, and $\sqrt{r}\left\|\hat{\Sigma}_{\hat{\nabla}, \hat{\underline{\Sigma}}_{r}}-\Sigma_{\nabla, \underline{\nabla}_{r}}\right\|$ tend to zero in probability as $n \rightarrow \infty$ when $r=o\left(n^{1 / 3}\right)$.

Proof. We first show that the replacement of the unknown initial values $\left\{X_{u}, u \leq 0\right\}$ by zero is asymptotically unimportant. Let $\hat{\Sigma}_{\underline{\Xi}_{r, n}}$ be the matrix obtained by replacing $e_{t}\left(\theta_{n}^{*}\right)$ by $\epsilon_{t}\left(\theta_{n}^{*}\right)$ in $\hat{\Sigma}_{\hat{\underline{V}}_{r}}$. Because $\epsilon_{t}(\theta)$ and their 
derivatives have ARMA representations (see Section 3), it is easy to show that

$$
\sup _{\theta \in \Theta^{*}}\left|e_{t}(\theta)-\epsilon_{t}(\theta)\right| \leq K \rho^{t}, \quad \sup _{\theta \in \Theta^{*}}\left\|\frac{\partial}{\partial \theta} e_{t}(\theta)-\frac{\partial}{\partial \theta} \epsilon_{t}(\theta)\right\| \leq K \rho^{t} .
$$

It can be deduced that $\left\|\hat{\Sigma}_{\hat{\underline{\underline{V}}}_{r}}-\hat{\Sigma}_{\underline{\underline{\nabla}}_{r, n}}\right\|=O_{P}\left(r n^{-1}\right)$. We thus have

$$
\sqrt{r}\left\|\hat{\Sigma}_{\hat{\underline{\nabla}}_{r}}-\hat{\Sigma}_{\underline{\nabla}_{r, n}}\right\|=o_{P}(1) .
$$

Taylor expansions around $\theta^{*}$ yield

$$
\left|\epsilon_{t}\left(\theta_{n}^{*}\right)-\epsilon_{t}\left(\theta^{*}\right)\right| \leq r_{t}\left\|\theta_{n}^{*}-\theta^{*}\right\|, \quad\left|\frac{\partial \epsilon_{t}\left(\theta_{n}^{*}\right)}{\partial \theta_{m}}-\frac{\partial \epsilon_{t}\left(\theta^{*}\right)}{\partial \theta_{m}}\right| \leq s_{t}\left\|\theta_{n}^{*}-\theta^{*}\right\|
$$

with $r_{t}=\left\|\frac{\partial}{\partial \theta^{\prime}} \epsilon_{t}(\bar{\theta})\right\|, s_{t}=\left\|\frac{\partial^{2}}{\partial \theta^{\prime} \partial \theta_{m}} \epsilon_{t}(\underline{\theta})\right\|$ where $\bar{\theta}$ and $\underline{\theta}$ are between $\theta_{n}^{*}$ and $\theta^{*}$. Define $Z_{t}$ as in the proof of Lemma 3 , and let $Z_{t, n}$ be obtained by replacing $\nabla_{t}(m)$ by $\nabla_{t, n}(m)=\epsilon_{t}\left(\theta_{n}^{*}\right) \partial \epsilon_{t}\left(\theta_{n}^{*}\right) / \partial \theta_{m}$ in $Z_{t}$. We have

$$
\begin{aligned}
\left|\nabla_{t}(m)-\nabla_{t, n}(m)\right| & \leq r_{t}\left\|\theta_{n}^{*}-\theta^{*}\right\|\left|\frac{\partial}{\partial \theta_{m}} \epsilon_{t}\left(\theta^{*}\right)\right|+s_{t}\left\|\theta_{n}^{*}-\theta^{*}\right\|\left|\epsilon_{t}\left(\theta_{n}^{*}\right)\right| \\
& :=\left\|\theta_{n}^{*}-\theta^{*}\right\| d_{t, n, m},
\end{aligned}
$$

and thus

$$
\left|Z_{t}-Z_{t, n}\right| \leq\left\|\theta_{n}^{*}-\theta^{*}\right\| D_{t, n, m_{1}, m_{2}, r_{1}, r_{2}},
$$

where

$$
D_{t, n, m_{1}, m_{2}, r_{1}, r_{2}}=\left|d_{t-r_{1}, n, m_{1}} \nabla_{t-r_{2}}\left(m_{2}\right)\right|+\left|\nabla_{t-r_{1}, n}\left(m_{1}\right) d_{t-r_{2}, n, m_{2}}\right| .
$$

Note that $E\left|D_{t, n, m_{1}, m_{2}, r_{1}, r_{2}}\right| \leq K$ for some constant $K$ independent of $n, r_{1}, r_{2}, m_{1}$ and $m_{2}$. Thus

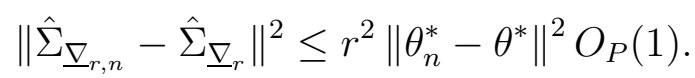

Since $\left\|\theta_{n}^{*}-\theta^{*}\right\|=O_{P}\left(n^{-1 / 2}\right)$, we obtain for $r=o\left(n^{1 / 3}\right)$

$$
\sqrt{r}\left\|\hat{\Sigma}_{\underline{\nabla}_{r, n}}-\hat{\Sigma}_{\underline{\nabla}_{r}}\right\|=o_{P}(1) .
$$

By Lemma $3,(24)$ and (25) show that $\sqrt{r}\left\|\hat{\Sigma}_{\hat{\underline{\Sigma}}_{r}}-\Sigma_{\underline{\underline{V}}_{r}}\right\|=o_{P}(1)$. The other results are obtained similarly.

Write $\underline{A}_{r}^{*}=\left(A_{1} \cdots A_{r}\right)$ where the $A_{i}$ 's are defined by (13). 
Lemma 5. Under the assumptions of Theorem A.1,

$$
\sqrt{r}\left\|\underline{A}_{r}^{*}-\underline{A}_{r}\right\| \rightarrow 0
$$

as $r \rightarrow \infty$.

Proof. Recall that by (13) and (15)

$$
\nabla_{t}=\underline{A}_{r} \underline{\nabla}_{r, t}+u_{r, t}=\underline{A}_{r}^{*} \underline{\nabla}_{r, t}+\sum_{i=r+1}^{\infty} A_{i} \nabla_{t-i}+u_{t}:=\underline{A}_{r}^{*} \underline{\nabla}_{r, t}+u_{r, t}^{*} .
$$

Hence, using the orthogonality conditions in (13) and (15)

$$
\underline{A}_{r}^{*}-\underline{A}_{r}=-\Sigma_{u_{r}^{*}, \underline{\nabla}_{r}} \Sigma_{\underline{\nabla}_{r}}^{-1}
$$

where $\Sigma_{u_{r}^{*}, \underline{\nabla}_{r}}=E u_{r, t}^{*} \underline{\nabla}_{r, t}^{\prime}$. Using arguments and notations of the proof of Lemma 2, there exists a constant $K_{2}$ independent of $s$ and $m_{1}, m_{2}$ such that

$$
E\left|\nabla_{1}\left(m_{1}\right) \nabla_{1+s}\left(m_{2}\right)\right| \leq K^{4} \sum_{h_{1}, \ldots, h_{4}=0}^{\infty} \rho^{h_{1}+\cdots+h_{4}}\left\|\epsilon_{1}\right\|_{4}^{4} \leq K_{2} .
$$

By the Cauchy-Schwarz inequality and (22), we then have

$$
\left\|\operatorname{Cov}\left(\nabla_{t-r-h}, \underline{\nabla}_{r, t}\right)\right\| \leq K_{2} r^{1 / 2}(p+q) .
$$

Thus,

$$
\begin{aligned}
\left\|\Sigma_{u_{r}^{*}, \underline{\nabla}_{r}}\right\| & =\left\|\sum_{i=r+1}^{\infty} A_{i} E \nabla_{t-i} \underline{\nabla}_{r, t}^{\prime}\right\| \leq \sum_{h=1}^{\infty}\left\|A_{r+h}\right\|\left\|\operatorname{Cov}\left(\nabla_{t-r-h}, \underline{\nabla}_{r, t}\right)\right\| \\
& =O(1) r^{1 / 2} \sum_{h=1}^{\infty}\left\|A_{r+h}\right\| .
\end{aligned}
$$

Note that the assumption $\left\|A_{i}\right\|=o\left(i^{-2}\right)$ entails $r \sum_{h=1}^{\infty}\left\|A_{r+h}\right\|=o(1)$ as $r \rightarrow \infty$. The lemma therefore follows from (26), (27) and Lemma 1.

The following lemma is similar to Lemma 3 in Berk (1974).

Lemma 6. Under the assumptions of Theorem A.1,

$$
\sqrt{r}\left\|\hat{\Sigma}_{\underline{\hat{\underline{V}}}_{r}^{-1}}^{-1} \Sigma_{\underline{\nabla}_{r}}^{-1}\right\|=o_{P}(1)
$$

as $n \rightarrow \infty$ when $r=o\left(n^{1 / 3}\right)$ and $r \rightarrow \infty$. 
Proof. We have

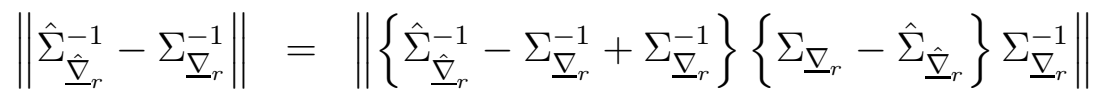

$$
\begin{aligned}
& \leq\left(\left\|\hat{\Sigma}_{\underline{\hat{\underline{V}}}_{r}^{-1}}^{-} \Sigma_{\underline{\nabla}_{r}}^{-1}\right\|+\left\|\Sigma_{\underline{\nabla}_{r}^{-1}}\right\|\right)\left\|\hat{\Sigma}_{\hat{\underline{\underline{V}}}_{r}}-\Sigma_{\underline{\Xi}_{r}}\right\|\left\|\Sigma_{\underline{\nabla}_{r}}^{-1}\right\| .
\end{aligned}
$$

Iterating this inequality, we obtain

$$
\left\|\hat{\Sigma}_{\hat{\underline{\underline{V}}}_{r}^{-1}}^{-1} \Sigma_{\underline{\nabla}_{r}}^{-1}\right\| \leq\left\|\Sigma_{\underline{\nabla}_{r}}^{-1}\right\| \sum_{i=1}^{\infty}\left\|\hat{\Sigma}_{\underline{\underline{\underline{V}}}_{r}}-\Sigma_{\underline{\nabla}_{r}}\right\|^{i}\left\|\Sigma_{\underline{\nabla}_{r}}^{-1}\right\|^{i} .
$$

Thus, for every $\varepsilon>0$,

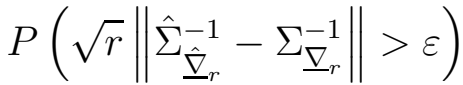

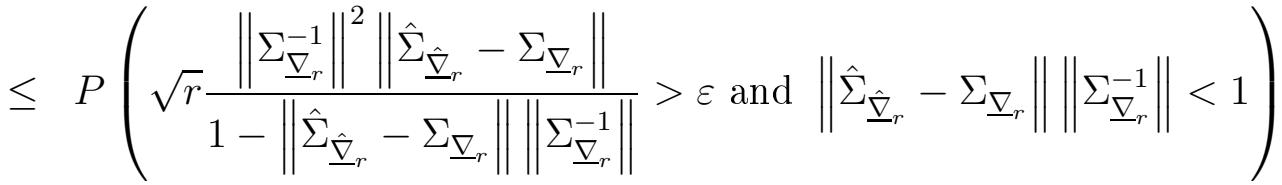

$$
\begin{aligned}
& +P\left(\sqrt{r}\left\|\hat{\Sigma}_{\hat{\underline{\underline{V}}}_{r}}-\Sigma_{\underline{\nabla}_{r}}\right\|\left\|\Sigma_{\underline{\nabla}_{r}^{-1}}\right\| \geq 1\right) \\
& \leq P\left(\sqrt{r}\left\|\hat{\Sigma}_{\hat{\underline{\nabla}}_{r}}-\Sigma_{\underline{\nabla}_{r}}\right\|>\frac{\varepsilon}{\left\|\Sigma_{\underline{\nabla}_{r}}^{-1}\right\|^{2}+\varepsilon r^{-1 / 2}\left\|\Sigma_{\underline{\nabla}_{r}}^{-1}\right\|}\right) \\
& +P\left(\sqrt{r}\left\|\hat{\Sigma}_{\hat{\underline{\underline{\Xi}}}_{r}}-\Sigma_{{\underline{\Xi_{r}}}_{r}}\right\| \geq\left\|\Sigma_{\underline{\underline{\nabla}}_{r}}^{-1}\right\|^{-1}\right)=o(1)
\end{aligned}
$$

by Lemmas 3 and 1. This establishes Lemma 6 .

Lemma 7. Under the assumptions of Theorem A.1,

$$
\sqrt{r}\left\|\underline{\hat{A}}_{r}-\underline{A}_{r}\right\|=o_{P}(1)
$$

as $r \rightarrow \infty$ and $r=o\left(n^{1 / 3}\right)$.

Proof. By the triangle inequality and Lemmas 1 and 6, we have

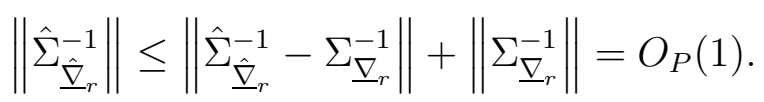


Note that the orthogonality conditions in (15) entail that $\underline{A}_{r}=\Sigma_{\nabla, \underline{\nabla}_{r}} \Sigma_{\underline{\nabla}_{r}}^{-1}$. By Lemmas 1, 3, 6, and (28), we then have

$$
\begin{aligned}
& \sqrt{r}\left\|\underline{\hat{A}}_{r}-\underline{A}_{r}\right\|=\sqrt{r} \| \hat{\Sigma}_{\hat{\nabla}, \hat{\Sigma}_{r}} \hat{\Sigma}_{\underline{\hat{\Sigma}}_{r}^{-1}}^{-\Sigma_{\nabla, \underline{\nabla}_{r}} \Sigma_{\underline{\nabla}_{r}}^{-1} \|}
\end{aligned}
$$

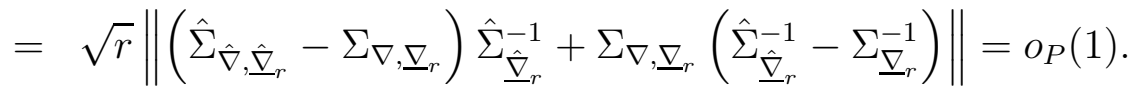

Proof of Theorem A.1. In view of (14), it suffices to show that $\hat{\mathcal{A}}_{r}(1) \rightarrow$ $\mathcal{A}(1)$ and $\hat{\Sigma}_{u_{r}} \rightarrow \Sigma_{u}$ in probability. Let the $r \times 1$ vector $\mathbf{1}_{r}=(1, \ldots, 1)^{\prime}$ and the $r(p+q) \times(p+q)$ matrix $\mathbf{E}_{r}=\mathbb{I}_{p+q} \otimes \mathbf{1}_{r}$, where $\otimes$ denotes the matrix Kronecker product and $\mathbb{I}_{d}$ the $d \times d$ identity matrix. Using (22), and Lemmas 5,7 , we obtain

$$
\begin{aligned}
\left\|\hat{\mathcal{A}}_{r}(1)-\mathcal{A}(1)\right\| & \leq\left\|\sum_{i=1}^{r} \hat{A}_{r, i}-A_{r, i}\right\|+\left\|\sum_{i=1}^{r} A_{r, i}-A_{i}\right\|+\left\|\sum_{i=r+1}^{\infty} A_{i}\right\| \\
& =\left\|\left(\underline{\hat{A}}_{r}-\underline{A}_{r}\right) \mathbf{E}_{r}\right\|+\left\|\left(\underline{A}_{r}^{*}-\underline{A}_{r}\right) \mathbf{E}_{r}\right\|+\left\|\sum_{i=r+1}^{\infty} A_{i}\right\| \\
& \leq \sqrt{p+q} \sqrt{r}\left\{\left\|\underline{\hat{A}}_{r}-\underline{A}_{r}\right\|+\left\|\underline{A}_{r}^{*}-\underline{A}_{r}\right\|\right\}+\left\|\sum_{i=r+1}^{\infty} A_{i}\right\| \\
& =o_{P}(1) .
\end{aligned}
$$

Now note that

$$
\hat{\Sigma}_{u_{r}}=\hat{\Sigma}_{\hat{\nabla}}-\underline{\hat{A}}_{r} \hat{\Sigma}_{\hat{\nabla}, \hat{\nabla}_{r}}^{\prime}
$$

and, by (13)

$$
\begin{aligned}
\Sigma_{u} & =E u_{t} u_{t}^{\prime}=E u_{t} \nabla_{t}^{\prime}=E\left\{\left(\nabla_{t}-\sum_{i=1}^{\infty} A_{i} \nabla_{t-i}\right) \nabla_{t}^{\prime}\right\} \\
& =\Sigma_{\nabla}-\sum_{i=1}^{\infty} A_{i} E \nabla_{t-i} \nabla_{t}^{\prime}=\Sigma_{\nabla}-\underline{A}_{r}^{*} \Sigma_{\nabla, \underline{\nabla}_{r}}^{\prime}-\sum_{i=r+1}^{\infty} A_{i} E \nabla_{t-i} \nabla_{t}^{\prime} .
\end{aligned}
$$


Thus,

$$
\begin{aligned}
& \left\|\hat{\Sigma}_{u_{r}}-\Sigma_{u}\right\|=\| \hat{\Sigma}_{\hat{\nabla}}-\Sigma_{\nabla}-\left(\underline{\hat{A}}_{r}-\underline{A}_{r}^{*}\right) \hat{\Sigma}_{\hat{\nabla}, \hat{\underline{V}}_{r}}^{\prime} \\
& -\underline{A}_{r}^{*}\left(\hat{\Sigma}_{\hat{\nabla}, \hat{\underline{\Sigma}}_{r}}^{\prime}-\Sigma_{\nabla, \underline{\nabla}_{r}}^{\prime}\right)+\sum_{i=r+1}^{\infty} A_{i} E \nabla_{t-i} \nabla_{t}^{\prime} \| \\
& \leq\left\|\hat{\Sigma}_{\hat{\nabla}}-\Sigma_{\nabla}\right\|+\left\|\left(\underline{\hat{A}}_{r}-\underline{A}_{r}^{*}\right)\left(\hat{\Sigma}_{\hat{\nabla}, \underline{\hat{E}}_{r}}^{\prime}-\Sigma_{\nabla, \underline{\nabla}_{r}}^{\prime}\right)\right\| \\
& +\left\|\left(\underline{\hat{A}}_{r}-\underline{A}_{r}^{*}\right) \Sigma_{\nabla, \underline{\nabla}_{r}}^{\prime}\right\|+\left\|\underline{A}_{r}^{*}\left(\hat{\Sigma}_{\hat{\nabla}, \hat{\underline{\nu}}_{r}}^{\prime}-\Sigma_{\nabla, \underline{\nabla}_{r}}^{\prime}\right)\right\| \\
& +\left\|\sum_{i=r+1}^{\infty} A_{i} E \nabla_{t-i} \nabla_{t}^{\prime}\right\| \text {. }
\end{aligned}
$$

In the right-hand side of this inequality, the first norm is $o_{P}(1)$ by Lemma 3 . By Lemmas 5 and 7, we have $\left\|\underline{\hat{A}}_{r}-\underline{A}_{r}^{*}\right\|=o_{p}\left(r^{-1 / 2}\right)=o_{p}(1)$, and by Lemma 3 , $\left\|\hat{\Sigma}_{\hat{\nabla}_{, \hat{\nabla}_{r}}}^{\prime}-\Sigma_{\nabla, \underline{\nabla}_{r}}^{\prime}\right\|=o_{p}\left(r^{-1 / 2}\right)=o_{p}(1)$. Therefore the second norm in the righthand side of (29) tends to zero in probability. The third norm tends to zero in probability because $\left\|\underline{\hat{A}}_{r}-\underline{A}_{r}^{*}\right\|=o_{p}(1)$ and, by Lemma $1,\left\|\Sigma_{\nabla, \underline{\nabla}_{r}}^{\prime}\right\|=O(1)$. The fourth norm tends to zero in probability because, in view of Lemma 3, $\left\|\hat{\Sigma}_{\hat{\nabla}, \hat{\nabla}_{r}}^{\prime}-\Sigma_{\nabla, \underline{\nabla}_{r}}^{\prime}\right\|=o_{p}(1)$, and, in view of $(22),\left\|\underline{A}_{r}^{*}\right\|^{2} \leq \sum_{i=1}^{\infty} \operatorname{Tr}\left(A_{i} A_{i}^{\prime}\right)<\infty$. Clearly, the last norm tends to zero, which completes the proof. 